
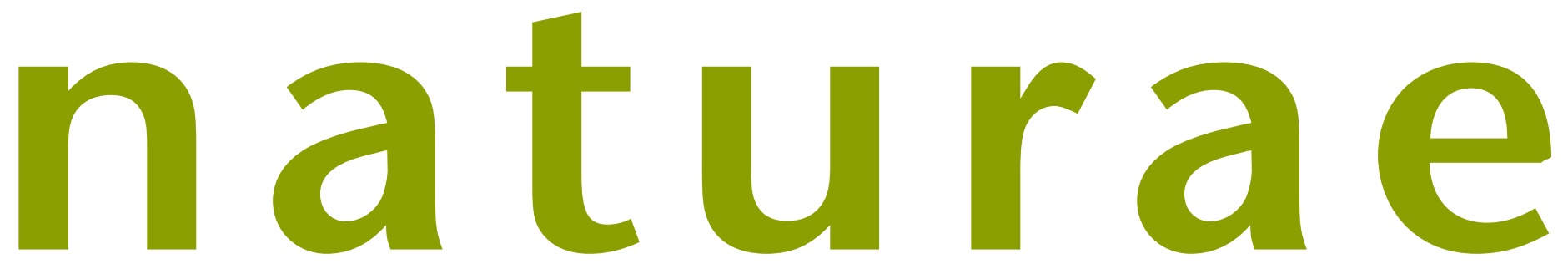

$2020 \cdot 11$

Apport de la bioacoustique à la systématique des oiseaux chanteurs: discussion à partir d'exemples chez les grimpereaux, merles dyals et gobemouches

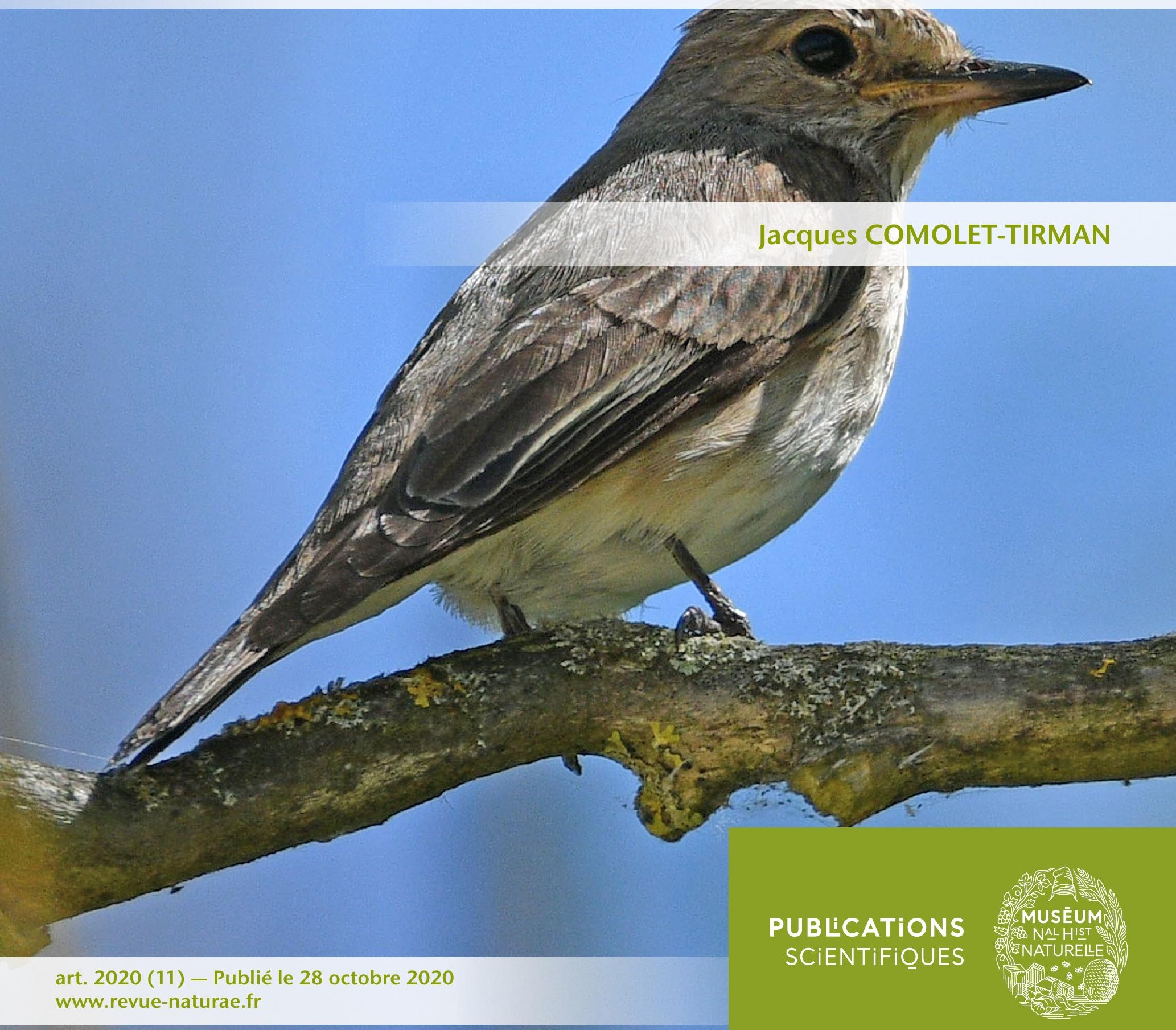


DiRECTEUR DE LA PUBLICATION / PUBLICATION DIRECTOR: Bruno David,

Président du Muséum national d'Histoire naturelle

RÉDACTEUR EN CHEF / EDITOR-IN-CHIEF: Jean-Philippe Siblet

ASSISTANTE DE RÉDACTION / ASSISTANT EDITOR: Sarah Figuet (naturae@mnhn.fr)

Mise EN PAGE / PAGE LAYOUT: Sarah Figuet

COMITÉ SCIENTIFIQUE / SCIENTIFIC BOARD:

Luc Abbadie (UPMC, Paris)

Luc Barbier (Parc naturel régional des caps et marais d'Opale, Colembert)

Aurélien Besnard (CEFE, Montpellier)

Vincent Boullet (Expert indépendant flore/végétation, Frugières-le-Pin)

Hervé Brustel (École d'ingénieurs de Purpan, Toulouse)

Patrick De Wever (MNHN, Paris)

Thierry Dutoit (UMR CNRS IMBE, Avignon)

Éric Feunteun (MNHN, Dinard)

Romain Garrouste (MNHN, Paris)

Grégoire Gautier (DRAAF Occitanie, Toulouse)

Olivier Gilg (Réserves naturelles de France, Dijon)

Frédéric Gosselin (Irstea, Nogent-sur-Vernisson)

Patrick Haffner (UMS PatriNat, Paris)

Frédéric Hendoux (MNHN, Paris)

Xavier Houard (OPIE, Guyancourt)

Isabelle Le Viol (MNHN, Concarneau)

Francis Meunier (Conservatoire d'espaces naturels - Hauts-de-France, Amiens)

Serge Muller (MNHN, Paris)

Francis Olivereau (DREAL Centre, Orléans)

Laurent Poncet (UMS PatriNat, Paris)

Nicolas Poulet (OFB, Vincennes)

Jean-Philippe Siblet (UMS PatriNat, Paris)

Laurent Tillon (ONF, Paris)

Julien Touroult (UMS PatriNat, Paris)

COUVERTURE / COVER:

Gobemouche gris Muscicapa striata striata (Pallas, 1764). Crédit photo: Jean-Philippe Siblet.

Naturae est une revue en flux continu publiée par les Publications scientifiques du Muséum, Paris Naturae is a fast track journal published by the Museum Science Press, Paris

Les Publications scientifiques du Muséum publient aussi / The Museum Science Press also publish:

Adansonia, Zoosystema, Anthropozoologica, European Journal of Taxonomy, Geodiversitas, Cryptogamie sous-sections Algologie, Bryologie, Mycologie, Comptes Rendus Palevol.

Diffusion - Publications scientifiques Muséum national d'Histoire naturelle

CP $41-57$ rue Cuvier F-75231 Paris cedex 05 (France)

Tél.: 33 (0)1 40794805 / Fax: 33 (0)1 40793840

diff.pub@mnhn.fr / http://sciencepress.mnhn.fr

(C) Publications scientifiques du Muséum national d'Histoire naturelle, Paris, 2020

ISSN (électronique / electronic): 1638-9387 


\title{
Apport de la bioacoustique à la systématique des oiseaux chanteurs: discussion à partir d'exemples chez les grimpereaux, merles dyals et gobemouches
}

\author{
Jacques COMOLET-TIRMAN \\ UMS PatriNat (OFB-CNRS-MNHN), \\ 57 rue Cuvier, boîte postale 41, F-75231 Paris cedex 05 (France) \\ jacques.comolet-tirman@ofb.gouv.fr
}

Soumis le 18 décembre 2019 | Accepté le 20 mai 2020 | Publié le 28 octobre 2020

\section{MOTS CLÉS \\ Passereaux, \\ chant, \\ Certhia,
Copsychus, \\ Muscicapa \\ systématique,} critères de Tobias.

\begin{abstract}
Comolet-Tirman J. 2020. -Apport de la bioacoustique à la systématique des oiseaux chanteurs: discussion à partir d'exemples chez les grimpereaux, merles dyals et gobemouches. Naturae 2020 (11): 165-184. https://doi.org/10.5852/ naturae2020a11

\section{RÉSUMÉ}

Cet article fait le point sur les possibilités d'utilisation des critères acoustiques en taxonomie des passereaux, en complément des critères morphologiques et moléculaires. Le premier exemple fait référence au Grimpereau des bois Certhia familiaris Linnaeus, 1758 et au Grimpereau des jardins Certhia brachydactyla C. L. Brehm, 1820, deux espèces distinguées de longue date mais dont les caractéristiques vocales ont récemment été quantifiées selon une approche standardisée basée sur la statistique d de Cohen. Un deuxième exemple est consacré au Merle dyal des Philippines Copsychus mindanensis (Boddaert, 1783) récemment séparé du Merle dyal Copsychus saularis (Linnaeus, 1758) sur la base de divers critères parmi lesquels les différences de vocalisations. Le troisième exemple détaille les études en cours sur le Gobemouche gris Muscicapa striata (Pallas, 1764) ainsi que les formes de gobemouches insulaires tyrrhenica et balearica considérées précédemment comme ses sous-espèces sous les noms M. striata tyrrhenica Schiebel, 1910 et M. striata balearica von Jordans, 1913 et dont la taxonomie fait encore l'objet de débats: l'auteur tente d'évaluer les différences vocales entre ces trois taxons selon la même approche standardisée. Les chants nuptiaux et territoriaux ont été analysés séparément. Parmi les résultats, les différences entre striata et tyrrhenica sont qualifiées d'exceptionnelles pour au moins deux caractères.
\end{abstract}

\section{ABSTRACT}

Contribution of bioacoustics to the systematics of songbirds: discussion based on examples from the Treecreepers, Magpie-robins and Flycatchers

This article reviews the possibilities of using acoustic criteria in passerine taxonomy, in addition to morphological and molecular criteria. The first example refers to the Eurasian Treecreeper Certhia familiaris Linnaeus, 1758 and the Short-toed Treecreeper Certhia brachydactyla C. L. Brehm, 1820, two long-standing distinguished species whose vocal characteristics have recently been quantified using a standardized approach. A second example is devoted to the Philippine Magpie-Robin Copsychus mindanensis (Boddaert, 1783), which was recently separated from the Oriental Magpie-Robin Copsychus saularis (Linnaeus, 1758) on the basis of various criteria, including vocalization differences. The third example details the ongoing studies on the Spotted Flycatcher Muscicapa striata (Pallas, 1764) and the Mediterranean taxa tyrrhenica and balearica previously known as its subspecies under the names M. striata tyrrhenica Schiebel, 1910 and M. striata balearica von Jordans, 1913 and whose taxonomy is still under discussion: the author attempts to assess the vocal differences between these three taxa using the same standardized approach applied to spectral measures (maximum, minimum 
KEY WORDS

Passerines,

song,

Copsychus,

Muscicapa

systematics,

Tobias criteria. and peak frequencies, bandwith). Nuptial songs and territorial songs have been analysed separately, with emphasis on nuptial songs for which Cohen's $d$ statistics have been calculated for each pair of taxa and for each character. However no attempt at calculating Cohen's statistics for minimum frequency has been made due to limitations in the data set (very faint flycatcher sounds are often difficult to identify when similar frequencies occur in the background noise). No analysis of temporal measurements has been made, but the presence of abrupt frequency changes has been added as an additional character, shared by balearica and tyrrhenica. Among the results are the differences between striata and tyrrhenica, which are described as exceptional for at least two characters (maximum frequency and peak frequency).
De nombreuses découvertes restent à faire en bioacoustique. "Les sons animaux, qu'ils soient audibles ou non, les organes qui les produisent et ceux qui les reçoivent sont loin d'être tous décrits» (Sueur \& Boistel 1998). Certains sons émis à des fréquences peu ou pas audibles pour notre oreille pourraient faire partie des sons non décrits.

\section{PROUESSES VOCALES ET AUDITIVES}

Dans le meilleur des cas, l'oreille humaine est sensible aux sons dont les fréquences sont comprises entre 20 et $20000 \mathrm{~Hz}$. L'acuité auditive des Oiseaux serait du même ordre de grandeur mais un pouvoir de résolution temporelle nettement supérieur leur permettrait d'entendre avec une grande précision la fine structure temporelle de certains sons que nous ne percevons que de manière très imparfaite. Ainsi une part importante de certaines informations contenues dans les vocalisations des oiseaux pourrait nous être inaccessible (Dooling \& Prior 2017).

De même, l'appréciation du caractère musical du chant d'un oiseau par l'oreille humaine est limitée. Le chant du Serin cini Serinus serinus (Linnaeus, 1766) décrit comme un «babil très soutenu et pressé, cliquetant, grinçant» (Peterson et al. 1971) paraît beaucoup plus mélodieux si on l'analyse à vitesse réduite, ce qui met en évidence des subtilités inattendues et un phrasé mélodieux proche de celui d'une fauvette Sylvia sp. Sur son site internet (http://www.whybirdssing.com, dernière consultation le 19 août 2020), David Rothenberg illustre la musicalité du chant des oiseaux avec l'exemple des grives américaines Catharus guttatus (Pallas, 1811) (Grive solitaire) et Catharus fuscescens (Stephens, 1817) (Grive fauve). Leur chant est proposé à vitesse normale mais aussi à vitesse réduite, ce qui permet de mieux en apprécier l'intérêt musical. «Certains chants d'oiseaux familiers deviennent alors des phrases de toute beauté, certaines nuances subtiles devenant perceptibles».

\section{EXEMPLE D'UTILISATION DES VOCALISATIONS EN TAXONOMIE : LES CRITĖRES DE TOBIAS}

Quelle que soit leur musicalité, les vocalisations des Oiseaux peuvent s'avérer d'une importance cruciale dans le cadre des études taxonomiques. Dans le but de confirmer ou au contraire d'infirmer la validité du traitement comme espèces distinctes de certaines formes proches, Tobias (Tobias et al. 2010) a introduit une approche par critères (dits critères de Tobias) en incluant les vocalisations à côté d'autres paramètres biométriques ou de plumage. Il a cherché à quantifier les divergences vocales en analysant les chants d'une cinquantaine de paires d'espèces de passereaux et apparentés. Il s'est concentré sur les chants plutôt que sur les notes d'appel, car les chants ont tendance à influencer le choix du partenaire et ils jouent donc un rôle dans l'isolement reproductif des oiseaux.

Les enregistrements de chants ont été compilés à partir de diverses archives sonores. La structure du chant a ensuite été quantifiée à l'aide de diverses mesures temporelles et spectrales (sept paramètres principaux). Au cas par cas, d'autres caractères mesurables ont été aussi inclus (changement de rythme ou de fréquence, intervalle inter-notes, etc.). Lors de l'évaluation de la divergence des chants, seules des vocalisations analogues ont été comparées (il ne serait pas approprié de comparer le chant d'une espèce à un type de cri de l'autre espèce; des difficultés peuvent être rencontrées lors de l'examen d'espèces chez lesquelles plusieurs types de chants ont été décrits, comme c'est le cas pour la Mésange boréale Poecile montanus (Conrad, 1827)).

\section{LES CRITÈRES DE TOBIAS ET LA STATISTIQUE D DE COHEN}

La statistique de Cohen utilisée par Tobias et al. (2010) est une méthode complémentaire des tests statistiques classiques qui permettent de tester une hypothèse nulle pour un seuil donné de significativité. La statistique de Cohen ne repose pas sur un test mais permet de catégoriser la différence entre deux moyennes : pas de différence; faible; majeure; exceptionnelle, etc. Le lecteur trouvera tous les détails (formule mathématique, comment les seuils ont été fixés) dans Tobias et al. (2010).

Parmi les résultats, citons à titre d'exemple la divergence vocale entre les deux espèces européennes de grimpereaux, le Grimpereau des bois Certhia familiaris Linnaeus, 1758 (Fig. 1) et le Grimpereau des jardins Certhia brachydactyla C. L. Brehm, 1820, qui est qualifiée de majeure $(\mathrm{d}>5)$ selon Tobias et al. (2010), en particulier du fait de deux paramètres (durée moyenne d'une phrase et fréquence minimale émise au cours d'une phrase) comme le résume le Tableau 1. 


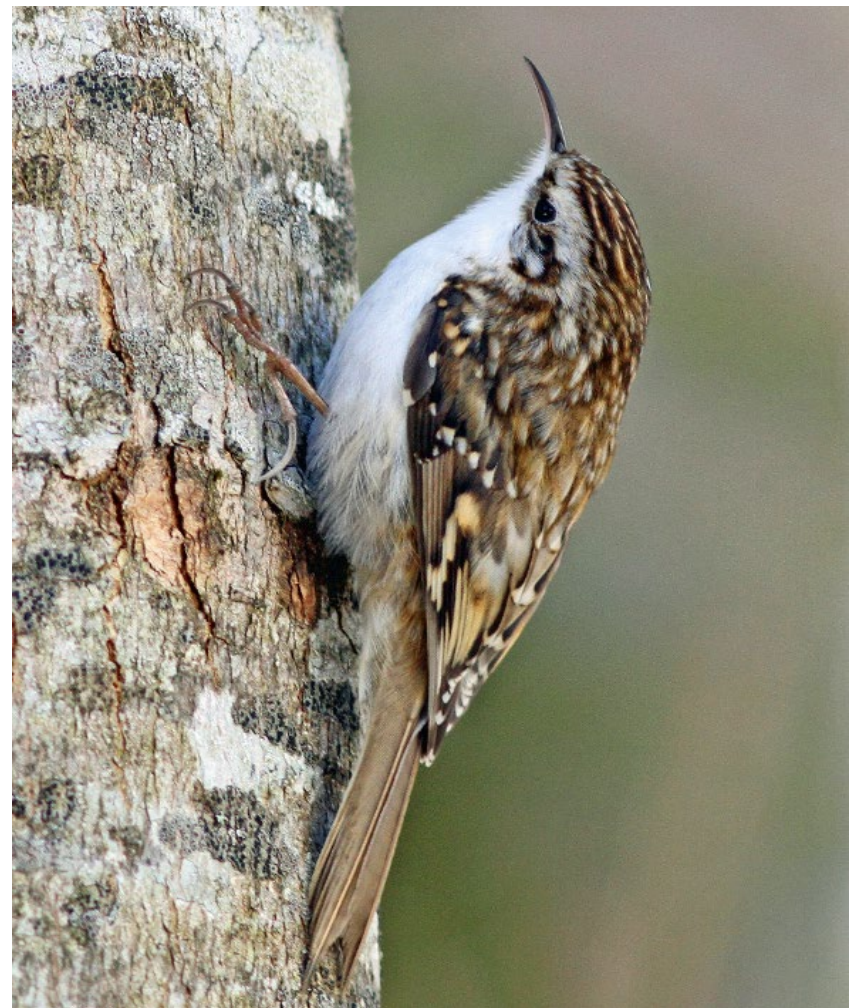

FIG. 1. - Les plumages des deux grimpereaux sont très similaires mais leurs chants diffèrent de façon marquée. Grimpereau des bois Certhia familiaris Linnaeus, 1758. Crédit photo: F. Jiguet.

Les chants de ces deux espèces utilisent des fréquences relativement élevées qui dépassent le plus souvent $5 \mathrm{kHz}$, nécessitant l'utilisation d'une échelle appropriée ( 0 à 8 , voire 0 à $10 \mathrm{kHz}$ ) lors de l'élaboration de sonagrammes.

Comme l'illustrent ces sonagrammes, une phrase de chant comporte un plus grand nombre de notes chez familiaris (Fig. 2: près d'une vingtaine, contre environ une demi-douzaine chez l'autre espèce), d'où une durée de phrase supérieure $(2,8 \mathrm{~s}$ contre à peine $1,2 \mathrm{~s}$ ). Les fréquences minimales sont un peu plus basses chez familiaris, passant en-dessous des $5 \mathrm{kHz}$. On pourrait ajouter que la phrase est globalement descendante chez familiaris, alors qu' elle est clairement montante, semblant interrogative, chez brachydactyla (Fig. 3). Cette approche trouve cependant ses limites du fait de cas bien documentés de chanteurs mixtes qui tendent à reprendre par imitation le chant de l'espèce jumelle.

\section{UNE APPLICATION À LARGE ÉCHELLE DES CRITĖRES DE TOBIAS}

Dans le cadre d'un ouvrage récent recensant les espèces d'oiseaux du monde entier (Del Hoyo \& Collar 2016), l'analyse quantitative des vocalisations a été pour la première fois introduite comme élément central du processus décisionnel taxonomique, en appliquant les critères de Tobias. Le but recherché était de réaliser un bilan aussi équitable que possible des caractères (biométrie, chant, plumage).
TABLEAU 1. - Tableau comparatif des chants de Certhia familiaris Linnaeus, 1758 et de Certhia brachydactyla C. L. Brehm, 1820 donnant les valeurs moyennes des principaux paramètres (d'après Tobias et al. 2010).

\begin{tabular}{lccc}
\hline $\begin{array}{c}\text { Principaux } \\
\text { paramètres }\end{array}$ & $\begin{array}{c}\text { Certhia familiaris } \\
\mathbf{n}=\mathbf{3}\end{array}$ & $\begin{array}{c}\text { Certhia brachy- } \\
\text { dactyla } \mathbf{n}=\mathbf{4}\end{array}$ & $\begin{array}{c}\text { Statistique } \\
\mathbf{d} \text { de } \\
\text { Cohen }\end{array}$ \\
\hline Durée & $2,424 \mathrm{~s}$ & $1,052 \mathrm{~s}$ & -5 \\
Nombre de & 16,083 & $\pm 0,184$ & \\
notes & $\pm 5,282$ & 5,75 & $-3,02$ \\
Rythme & 6,511 notes/s & 5,537 notes $/ \mathrm{s}$ & $-0,85$ \\
d'émission & $\pm 1,329$ & $\pm 0,995$ & \\
Fréquence & $3,059 \mathrm{kHz}$ & $4,569 \mathrm{kHz}$ & 7,42 \\
minimale & $\pm 0,125$ & $\pm 0,242$ & \\
Fréquence & $8,129 \mathrm{kHz}$ & $7,671 \mathrm{kHz}$ & $-1,39$ \\
maximale & $\pm 0,417$ & $\pm 0,254$ & \\
Fréquence & $5,951 \mathrm{kHz}$ & $5,413 \mathrm{kHz}$ & $-0,88$ \\
dominante & $\pm 0,594$ & $\pm 0,623$ & \\
Étendue & $5,07 \mathrm{kHz}$ & $3,103 \mathrm{kHz}$ & $-4,5$ \\
& $\pm 0,538$ & $\pm 0,356$ & \\
\hline
\end{tabular}

Dans plusieurs douzaines de cas, l'évaluation de la voix a été décisive pour décider s'il fallait élever les taxons au rang d'espèce. À titre d'exemple le Merle dyal des Philippines Copsychus mindanensis (Boddaert, 1783) (aussi appelé Shama des Philippines) a été séparé du Merle dyal Copsychus saularis (Linnaeus, 1758) (aussi appelé Shama dayal, Figs 4 et 5) sur la base de divers critères dont les vocalisations. Les résultats résumés de ces évaluations figurent dans l'ouvrage, et les résultats détaillés ont été mis à disposition ultérieurement sur internet (Boesman 2016).

Les chants de ces deux espèces utilisent des fréquences plus graves que celles des grimpereaux, de sorte qu'une échelle 0 à $6 \mathrm{kHz}$ peut convenir pour l'édition des sonagrammes. Le chant du Merle dyal des Philippines (Fig. 6) est caractérisé par des phrases plus longues et moins clairement délimitées. Les notes sont dans l'ensemble plus graves, ne dépassant pas $5 \mathrm{kHz}$, et la gamme de fréquence est réduite (selon Boesman [2016], "All stay below $5 \mathrm{kHz}$ and have a rather narrow frequency range»). Sur le sonagramme, un insecte stridule entre 3 et $4 \mathrm{~s}$ alors que l'oiseau émet des notes plutôt graves.

\section{ÉVALUATION DES DIVERGENCES VOCALES CHEZ LES GOBEMOUCHES MUSCICAPA}

Le Gobemouche gris Muscicapa striata (Pallas, 1764) est un petit Passereau insectivore largement répandu à travers l'Europe. Plusieurs sous-espèces ont été décrites notamment dans les îles méditerranéennes. L'élévation au rang d'espèce des formes insulaires du Gobemouche gris sous le nom de Muscicapa tyrrhenica a récemment été proposée (Pons et al. 2016, 2017) et acceptée par certaines autorités taxonomiques.

Cependant Del Hoyo \& Collar (2016) n’ont pas retenu cette proposition du fait de différences morphologiques peu prononcées et de différences vocales non quantifiées à l'époque, faute d'étude acoustique dédiée. 


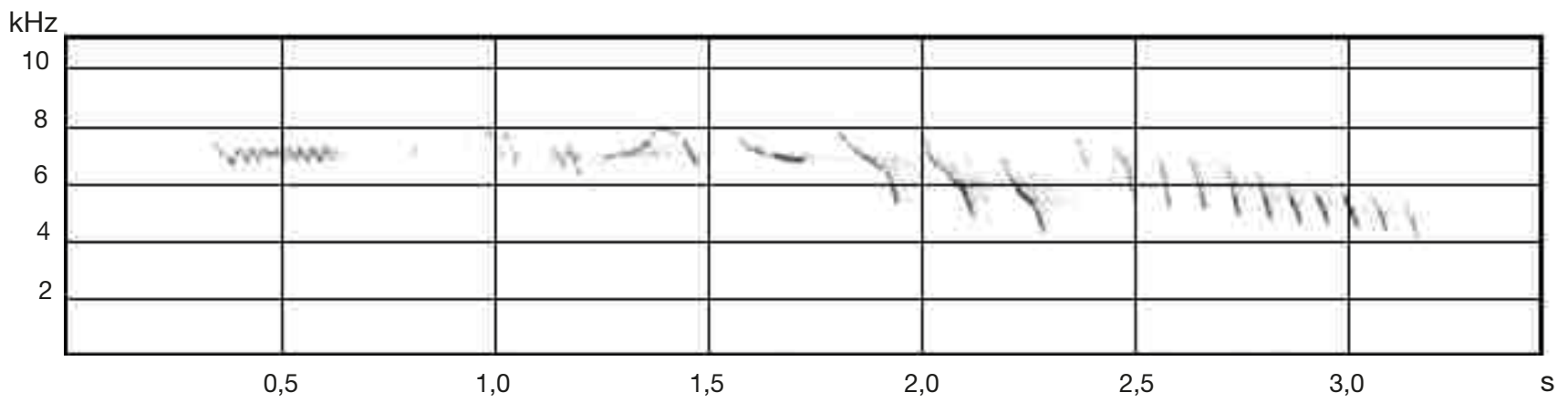

FIG. 2. - Une phrase de chant de Grimpereau des bois Certhia familiaris Linnaeus, 1758, Forêt de Compiègne, Oise, France, enregistrement de J. ComoletTirman (mai 1992). Son MNHN no MNHN-SO-2020-621.

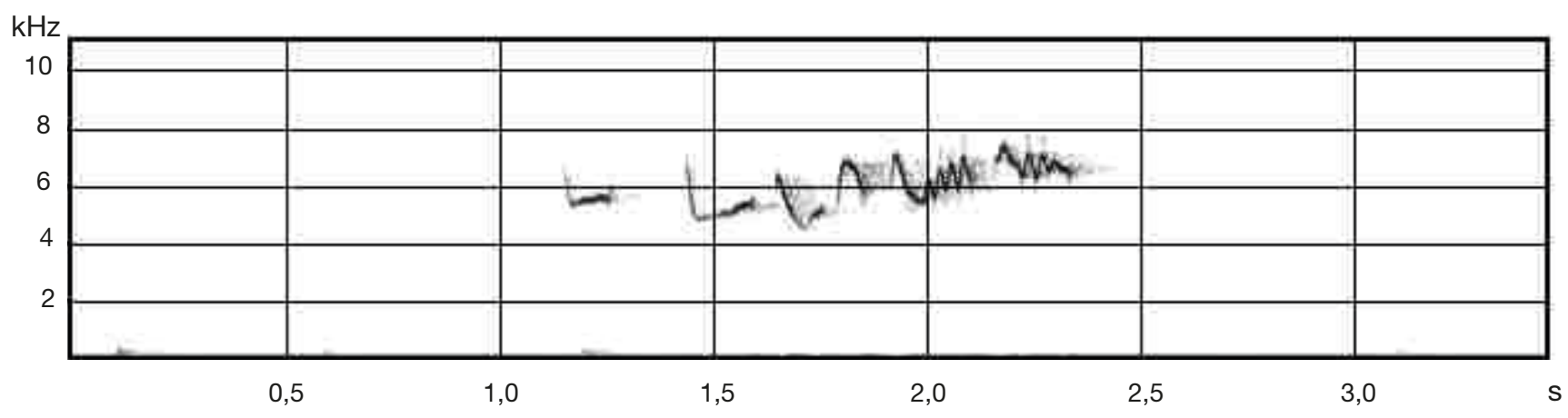

FIG. 3. - Une phrase de chant de Grimpereau des jardins Certhia brachydactyla C. L. Brehm, 1820, Forêt de Fontainebleau, Seine-et-Marne, France, enregistrement de J. Comolet-Tirman (mai 1989). Son MNHN no MNHN-SO-2020-622.

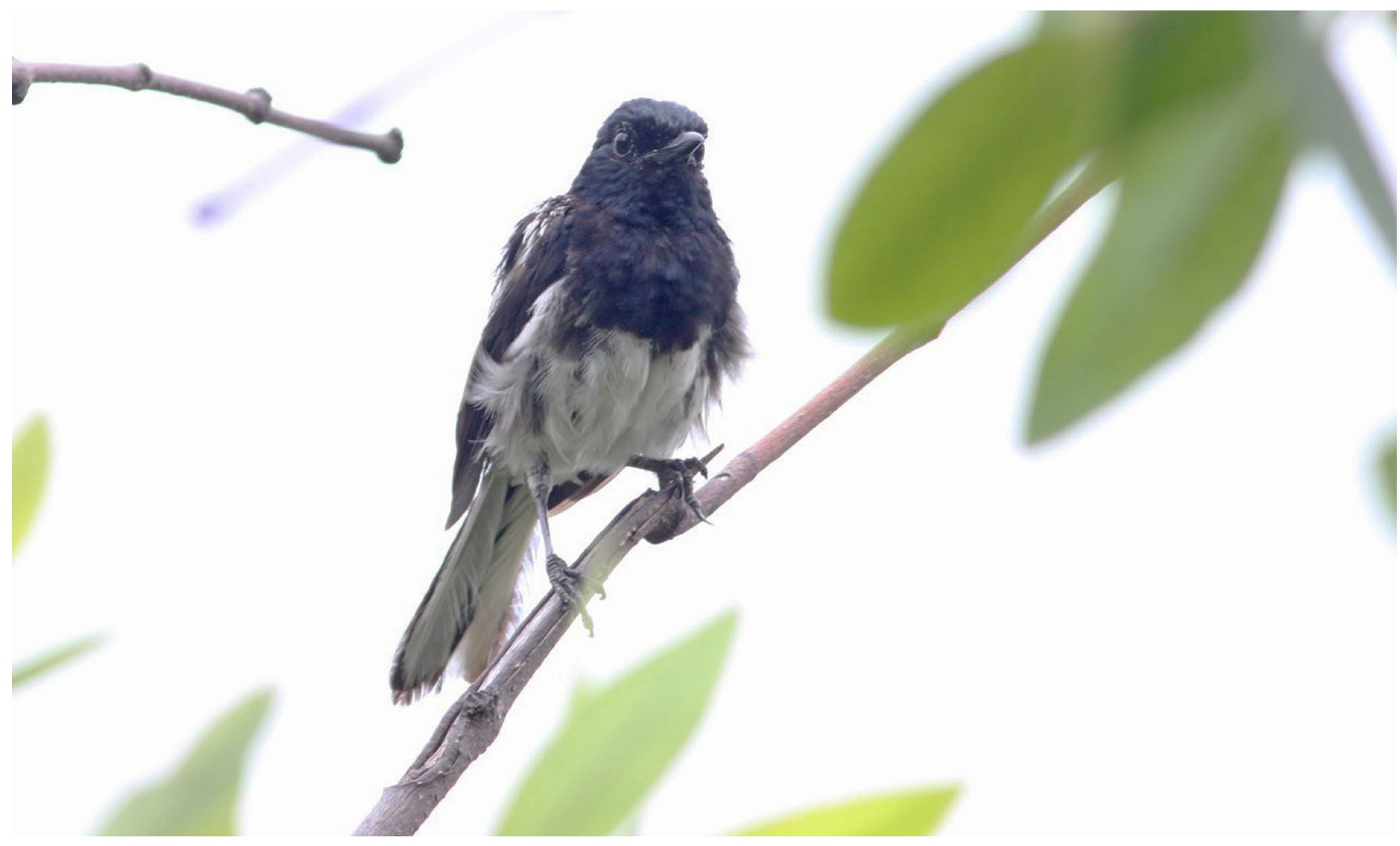

FIG. 4. - Merle dyal Copsychus saularis (Linnaeus, 1758), Cambodge, juillet 2015. crédit photo: J. Comolet-Tirman. 


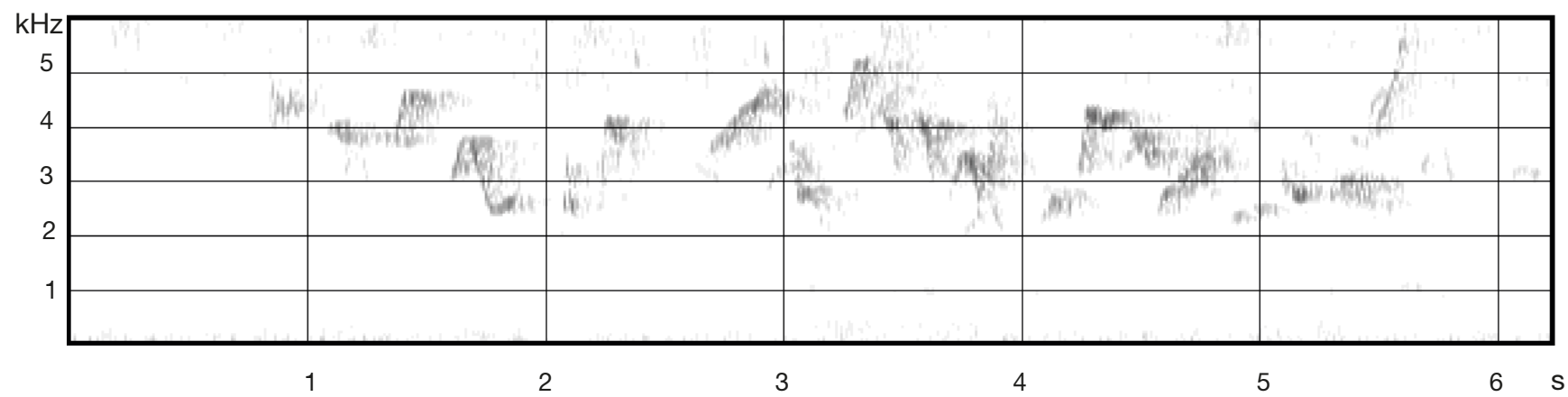

FIG. 5. - Une phrase de chant de Merle dyal Copsychus saularis (Linnaeus, 1758), Malaisie, enregistrement de J. Comolet-Tirman (juin 2006). Son MNHN no MNHN-SO-2020-623.

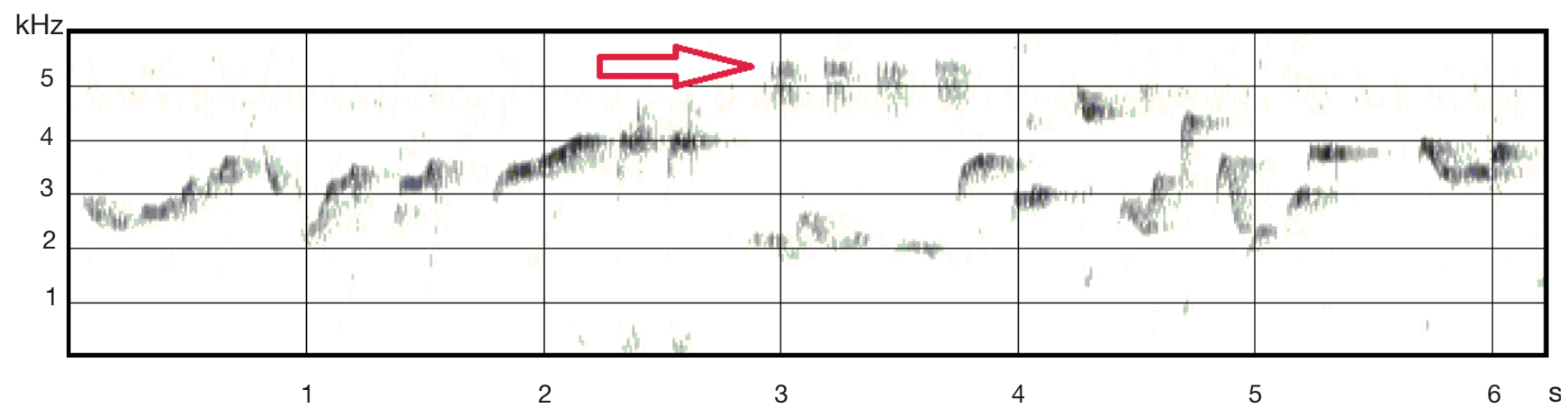

FIG. 6. - Chant de Merle dyal des Philippines Copsychus mindanensis (Boddaert, 1783), Leyte (Philippines), enregistrement de J. Comolet-Tirman (mars 2011). Les quatre motifs signalés par la flèche ne font pas partie du chant de l'oiseau (stridulation d'insecte). Son MNHN no MNHN-SO-2020-624.

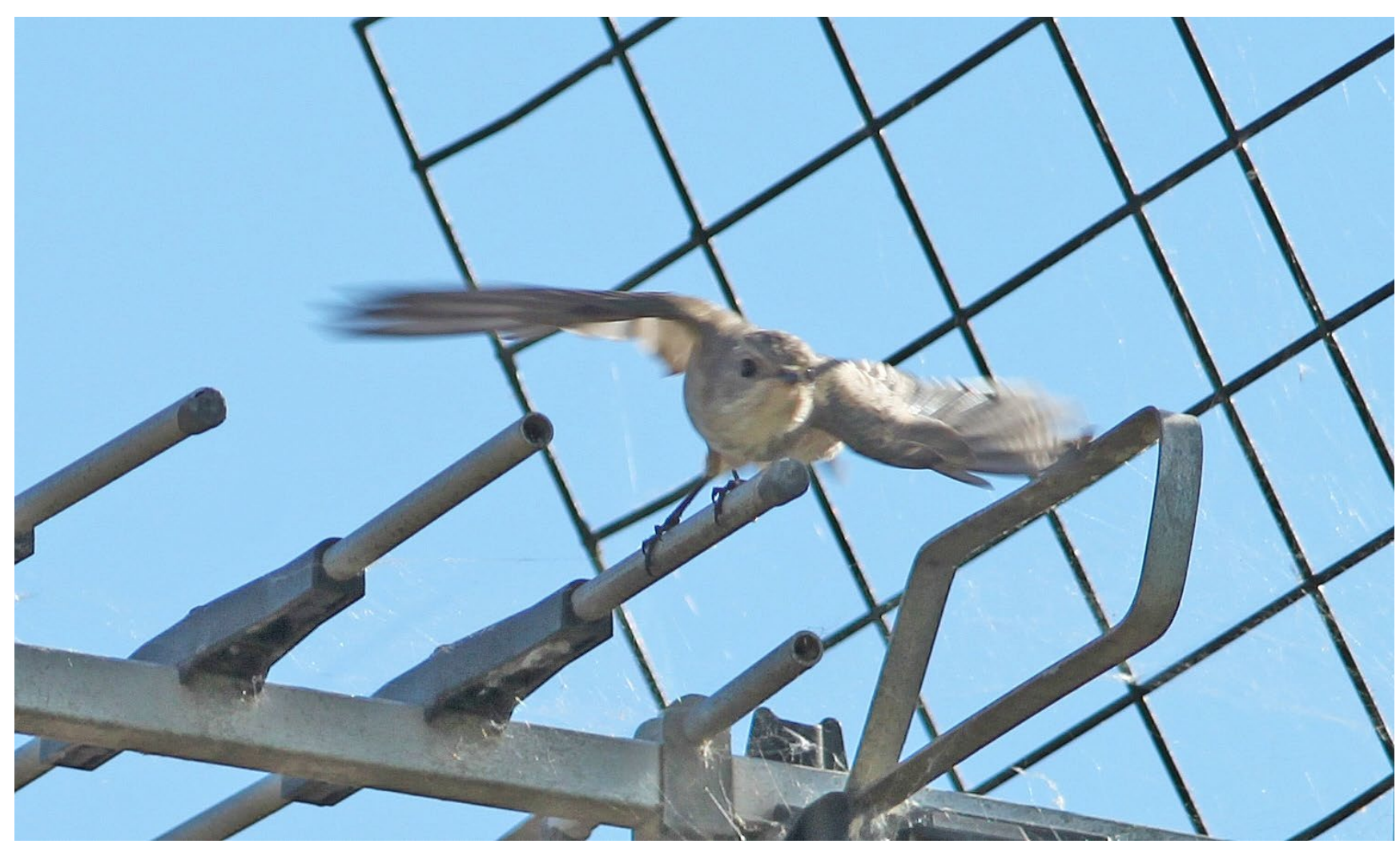

FIG. 7. - Gobemouche méditerranéen Muscicapa tyrrhenica tyrrhenica, Aleria (Haute-Corse), mai 2017. Crédit photo: J. Comolet-Tirman. 
TABLEAU 2. - Tableau comparatif des chants territoriaux de trois formes de gobemouches Muscicapa (parmi les chants territoriaux de tyrrhenica, trois proviennent de Corse et un de Sardaigne). La fréquence est exprimée en $\mathrm{kHz}$.

\begin{tabular}{|c|c|c|c|}
\hline Principaux paramètres & $\begin{array}{l}\text { striata }(n=8) \\
\text { Moy. } \pm \text { écart-type }\end{array}$ & $\begin{array}{l}\text { balearica }(n=3) \\
\text { Moy. } \pm \text { écart-type }\end{array}$ & $\begin{array}{l}\text { tyrrhenica }(n=4) \\
\text { Moy. } \pm \text { écart-type }\end{array}$ \\
\hline Fréquence minimale & $\begin{array}{l}3,62 \pm 1,18 \\
\operatorname{Min} 1,8 \operatorname{Max} 5\end{array}$ & $\begin{array}{c}6,06 \pm 0,11 \\
\operatorname{Min} 6 \operatorname{Max} 6,2\end{array}$ & $\begin{array}{c}\text { 4,88 } \pm 0,6 \\
\text { Min 4,05 Max } \\
5,5\end{array}$ \\
\hline Fréquence maximale & $\begin{array}{c}9,71 \pm 0,49 \\
\text { Min } 9 \operatorname{Max} 10,5\end{array}$ & $\begin{array}{c}12,1 \pm 1,83 \\
\text { Min } 10,1 \text { Max } \\
13,7\end{array}$ & $\begin{array}{c}18,11 \pm 1,79 \\
\operatorname{Min} 15,8 \operatorname{Max} 20\end{array}$ \\
\hline Fréquence dominante & $\begin{array}{c}7,32 \pm 0,24 \\
\text { Min 6,84 Max } \\
7,55\end{array}$ & $\begin{array}{c}7,09 \pm 0,49 \\
\text { Min } 6,59 \text { Max } \\
7,58\end{array}$ & $\begin{array}{c}7,14 \pm 0,33 \\
\operatorname{Min} 6,85 \text { Max } \\
7,62\end{array}$ \\
\hline Étendue moyenne & $\begin{array}{c}6,08 \pm 1,18 \\
\operatorname{Min} 4,5 \operatorname{Max} 7,4\end{array}$ & $\begin{array}{c}6,03 \pm 1,75 \\
\operatorname{Min} 4,1 \operatorname{Max} 7,5\end{array}$ & $\begin{array}{c}13,22 \pm 1,36 \\
\text { Min } 11,75 \text { Max } \\
15\end{array}$ \\
\hline Registre habituel & $\begin{array}{l}6-8 \text { parfois } 9 \text { motifs spéciaux } \\
\text { vers } 4 \text { ou } 5\end{array}$ & $\begin{array}{l}6-8 \text { brièvement jusque } \\
\text { vers } 10 \text { à } 12\end{array}$ & $\begin{array}{l}6-8 \text { brièvement jusque } \\
\text { vers } 15 \text { à } 18\end{array}$ \\
\hline $\begin{array}{l}\text { Sons variant de plusieurs } \mathrm{kHz} \\
\text { en moins de } 20 \mathrm{~ms}\end{array}$ & Absents & Réguliers mais écarts limités & Réguliers avec écarts importants \\
\hline
\end{tabular}

Afin de remédier à ces lacunes de connaissance, nous avons entrepris une étude des chants des formes insulaires tout d'abord en Corse (Fig. 7 ; Comolet-Tirman 2017) puis aux Baléares (Comolet-Tirman 2018). Le nombre d'individus enregistrés au cours de cette étude est relativement faible, mais depuis d'autres enregistrements réalisés par d'autres preneurs de son ont permis de confirmer les premiers résultats et certains d'entre eux ont pu être utilisés dans les nouvelles statistiques présentées ici (la liste des références sonores est donnée en Annexe 1 et des éléments plus détaillés sur les caractéristiques des chants individuels sont donnés en Annexes 2 et 3). L'étude du contexte comportemental des émissions sonores a permis de valider leur classification en trois types principaux : cris, chants territoriaux simples, chants nuptiaux complexes et rapides (émis dans un contexte particulier d'excitation à proximité d'un site potentiel de nidification, le plus souvent en présence de la femelle). Ces vocalisations ont ensuite pu être comparées à leur équivalent chez les oiseaux continentaux, là encore avec de nouveaux enregistrements disponibles. Dans le cadre de cette étude, seuls les chants seront comparés, selon la méthode définie par Tobias. Les cris ne semblent pas différer dans l'état actuel des connaissances, bien que l'étude des cris de migration pourrait réserver des surprises (Wroza 2019).

Les chants des gobemouches sont susceptibles de contenir des notes de fréquences très élevées. En conséquence une échelle de 0 à $20 \mathrm{kHz}$ a été nécessaire pour la représentation sur sonagrammes des différentes vocalisations. Les chants territoriaux sont émis sur un rythme lent qui ne semble guère différer entre les formes de gobemouches (de l'ordre de une note par seconde). Viganò (2015) a étudié le rythme d'émission des chants et n'a pas trouvé de différence significative entre tyrrhenica et striata. L'étude du rythme des chants nuptiaux des gobemouches ne sera pas abordée ici, mais d'autres paramètres du chant nuptial semblent discriminants.

La fréquence dominante émise dans les chants territoriaux (Tableau 2) ne diffère guère entre les différentes formes de gobemouches, elle est de l'ordre de $7 \mathrm{kHz}$ pour un registre habituel compris entre 6 et $8 \mathrm{kHz}$ (Fig. 8). Les chants territoriaux «insulaires» different de manière assez subtile, avec l'ajout d'une sorte de signature pouvant monter dans les aigus pour atteindre les $12 \mathrm{kHz}$ chez balearica (Fig. 9, son extrait de Chappuis 1976) et surtout $17 \mathrm{kHz}$ et parfois au-delà chez tyrrhenica (Fig. 10). Malgré tout l'étendue du registre est très similaire entre striata et balearica, en moyenne de $6 \mathrm{kHz}$. En revanche elle s'élève à plus du double chez tyrrhenica (précisions sur taille de l'échantillon, moyenne et écart-type au Tableau 2).

La fréquence dominante émise dans les chants nuptiaux (Tableau 3) diffère de façon importante entre les différentes formes de gobemouches. Alors qu'elle est de $7,4 \mathrm{kHz}$ chez striata, elle est d'environ $16,7 \mathrm{kHz}$ chez tyrrhenica (il faut cependant noter l'existence de plusieurs crêtes de fréquence souvent presque à égalité). Une position intermédiaire est occupée par balearica avec $11,6 \mathrm{kHz}$. Le registre habituel couvre environ $5 \mathrm{kHz}$ aussi bien chez striata ( 5 à $10 \mathrm{kHz}$ ) que chez balearica (7 à $12 \mathrm{kHz}$ ) mais il s'étend sur plus de $10 \mathrm{kHz}$ chez tyrrhenica (7 à 17 voire $18 \mathrm{kHz}$ ). Les chants nuptiaux de striata (Fig. 11) et balearica (Fig. 12) nous ont semblé assez semblables en termes de structure mais cette appréciation est peut-être biaisée par le faible nombre d'individus enregistrés et a été discutée (Viganò et al. 2019). Quoi qu'il en soit, les gobemouches insulaires diffèrent fortement des continentaux par leurs fréquences nettement plus élevées. Le chant nuptial de tyrrhenica en particulier (Fig. 13) diffère de façon radicale en raison de la présence de nombreux sons qui chutent rapidement depuis des fréquences extrêmement élevées. En effet, alors que les gobemouches continentaux atteignent tout au plus des fréquences comprises entre 10 et $11 \mathrm{kHz}$ et que ceux des Baléares atteignent voire dépassent la classe 12 à $13 \mathrm{kHz}$, les sons brefs et suraigus émis par tyrrhenica montent jusqu'à $18 \mathrm{kHz}$ et au-delà, frôlant les $20 \mathrm{kHz}$. Ils sont sans équivalent connu dans l'univers sonore des oiseaux et il s'agit probablement de la plus haute fréquence jamais documentée à ce jour dans le chant d'un passereau. 


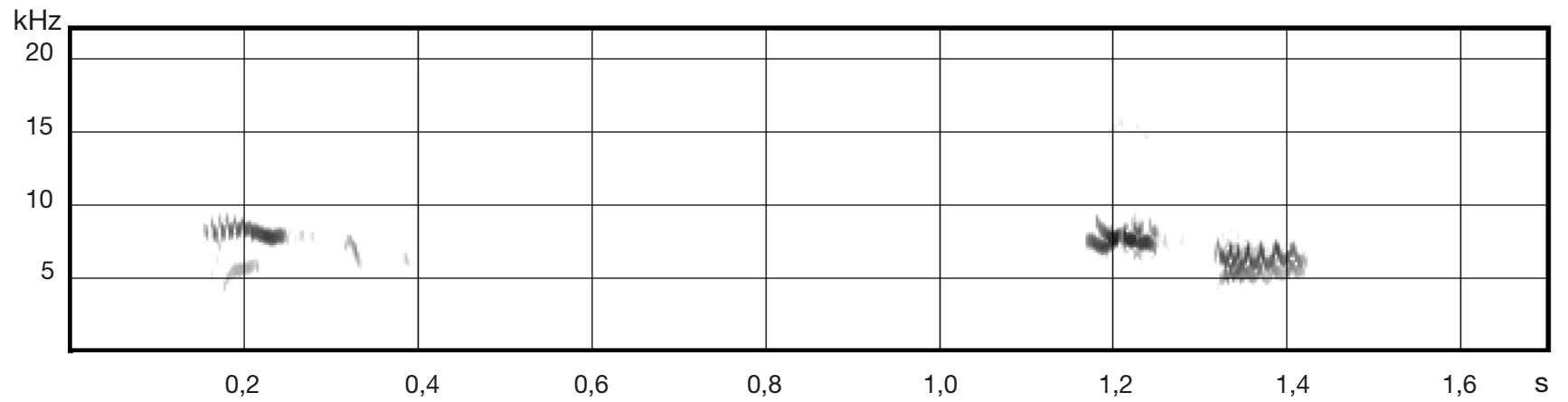

FIG. 8. - Chant territorial de Gobemouche gris Muscicapa striata striata, Fontainebleau (Seine-et-Marne, France), enregistrement de Julien Rochefort (mai 2013). Son MNHN no MNHN-SO-2020-620.

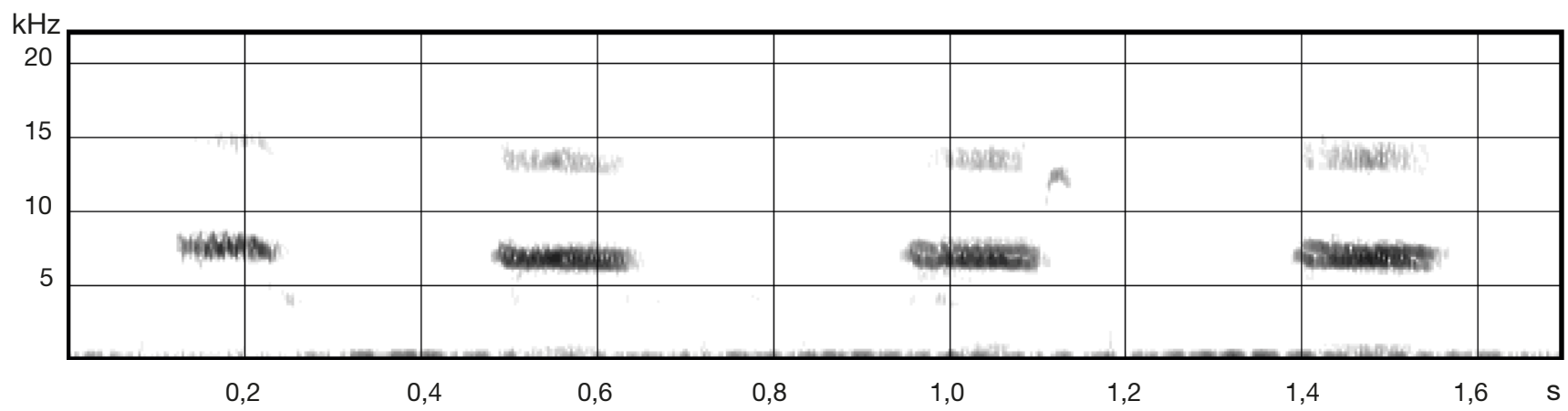

FIG. 9. - Chant territorial de Gobemouche méditerranéen Muscicapa tyrrhenica balearica, Majorque (Baléares, Espagne), enregistrement de Claude Chappuis (juin 1975). Les harmoniques sont bien visibles mais la troisième note est aussi suivie d'un élément de fréquence élevée qui n'est pas une harmonique mais bien un son fondamental.

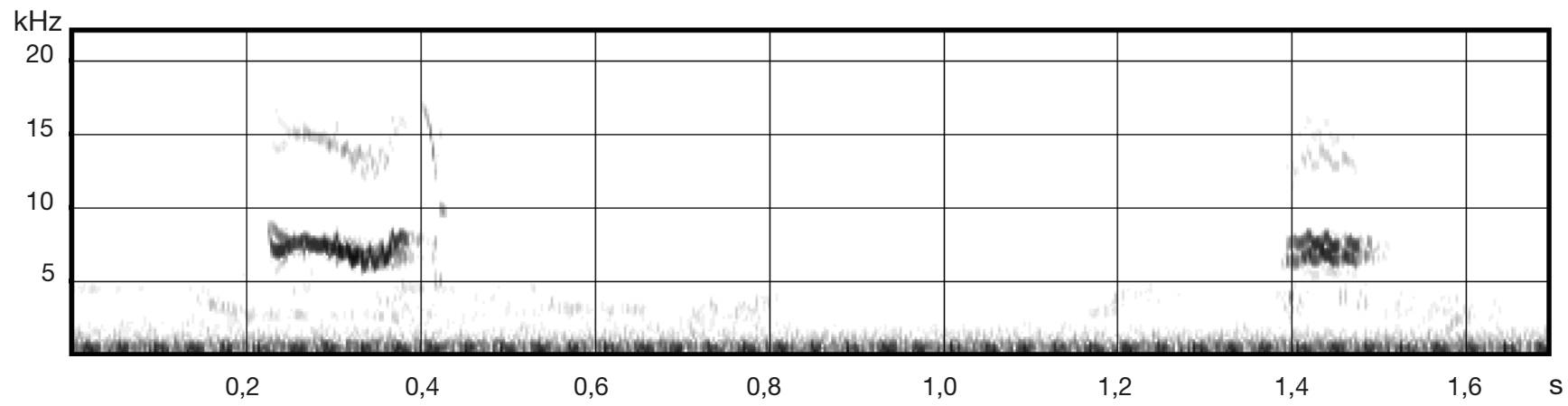

FIG. 10. - Chant territorial de Gobemouche méditerranéen Muscicapa tyrrhenica tyrrhenica, Pietracorbara (Haute-Corse, France) enregistrement de J. ComoletTirman (mai 2017). Son MNHN no MNHN-SO-2017-109. Les harmoniques sont bien visibles mais la première note est aussi suivie d'un élément de fréquence élevée qui n'est pas une harmonique mais bien un son fondamental.

Ces sons pourraient échapper en grande partie à l'oreille humaine et nécessitent d'être écoutés à vitesse réduite ou visualisés sur sonagrammes.

Les différences entre les chants nuptiaux ont été étudiées pour deux caractères à l'aide de la statistique d de Cohen (Tableau 4). Elles sont exceptionnelles (loin au-dessus des valeurs seuils) pour les chants de tyrrhenica versus striata, et majeures à exceptionnelles pour les autres paires de taxons. À travers leur comparaison de 58 paires d'espèces sympatriques, Tobias et al. (2010) ont trouvé huit paires d'espèces caractérisées par des différences vocales majeures $(\mathrm{d}>5)$ dont les grimpereaux Certhia sp. évoqués précédemment, mais aussi les mésanges Poecile montanus (Conrad, 1827) et $P$. palustris (Linnaeus, 1758), et seulement deux paires d'espèces caractérisées par des différences vocales exceptionnelles $(\mathrm{d}>10)$ : Empidonax alnorum Brewster, 1895 et E. traillii (Audubon, $1828)$ pour le nombre de notes $(\mathrm{d}=14,8)$ et le rythme d'émission $(\mathrm{d}=16,5)$, Pericrocotus brevirostris (Vigors, 1831) et P. ethologus Bangs \& Phillips, 1914 pour le rythme d'émission $(\mathrm{d}=-18)$. Si l'on s'intéresse aux fréquences maximales, les différences sont au mieux majeures $(\mathrm{d}>5)$ : Illadopsis fulvescens (Cassin, 1859) et I. puvelli (Salvadori, 1901) ( $\mathrm{d}=-6)$, Tyrannus verticalis Say, 1822 et T. vociferans Swainson, 1826 $(d=5,7)$. De même pour les fréquences dominantes avec 
TABLEAU 3. - Tableau comparatif des chants nuptiaux de trois formes de gobemouches Muscicapa (tous les chants nuptiaux de tyrrhenica proviennent de Corse).

\begin{tabular}{|c|c|c|c|}
\hline Principaux paramètres & striata $(n=4)$ & $\begin{array}{l}\text { balearica }(n=3) \\
\quad n=2 \text { pour fréquence } \min .\end{array}$ & $\begin{array}{l}\text { tyrrhenica }(n=4) \\
\quad n=3 \text { pour fréquence min. }\end{array}$ \\
\hline Fréquence minimale & $\begin{array}{c}1,49 \pm 0,39 \\
\operatorname{Min} 1,15 \operatorname{Max} 1,92\end{array}$ & $\begin{array}{l}4,82 \text { sous réserve } \\
\text { Min } 2,75 \text { Max } 6,9\end{array}$ & $\begin{array}{c}2,72 \pm 0,57 \\
\operatorname{Min} 2,07 \operatorname{Max} 3,4\end{array}$ \\
\hline Fréquence maximale & $\begin{array}{c}10,34 \pm 0,33 \\
\text { Min 10,07 Max 10,71 }\end{array}$ & $\begin{array}{c}12,7 \pm 0,69 \\
\text { Min } 12,25 \text { Max 13,5 }\end{array}$ & $\begin{array}{c}18,12 \pm 0,5 \\
\text { Min } 17 \operatorname{Max} 19\end{array}$ \\
\hline Fréquence dominante & $\begin{array}{c}7,4 \pm 0,32 \\
\operatorname{Min} 7,19 \operatorname{Max} 7,79\end{array}$ & $\begin{array}{c}11,63 \pm 0,88 \\
\text { Min 10,8 Max 12,56 }\end{array}$ & $\begin{array}{c}16,75 \pm-0,5 \\
\text { Min } 14,8 \text { Max } 17,88\end{array}$ \\
\hline Étendue moyenne & $\begin{array}{c}8,85 \pm 0,22 \\
\text { Min 8,67 Max 9,1 }\end{array}$ & $\begin{array}{l}\text { 7, } 49 \text { sous réserve } \\
\text { Min } 5,48 \text { Max } 9,5\end{array}$ & $\begin{array}{c}15,78 \pm 0,33 \\
\text { Min } 15,4 \operatorname{Max} 16\end{array}$ \\
\hline Registre habituel & 5 à 10 (étendue 5) & 7 à 12 (étendue 5) & 7 à 17 (étendue 10) \\
\hline $\begin{array}{l}\text { Sons variant de plusieurs } \mathrm{kHz} \text { en } \\
\text { moins de } 20 \mathrm{~ms}\end{array}$ & Absents & $\begin{array}{l}\text { Présents mais limités à environ } \\
5 \mathrm{kHz} \text { d'écart et régularité } \\
\text { à préciser }\end{array}$ & $\begin{array}{l}\text { Très réguliers, pouvant atteindre } \\
5 \text { à } 10 \mathrm{kHz} \text { d'écart }\end{array}$ \\
\hline
\end{tabular}

Criniger calurus (Cassin, 1856) et $C$.ndussumensis Reichenow, $1904(\mathrm{~d}=6,2)$. L'étude de la fréquence dominante permet de décrire le chant de balearica comme intermédiaire entre striata et tyrrhenica. Celle de la fréquence maximale suggère qu'il pourrait être légèrement plus proche des striata du continent que de tyrrhenica. Cependant seule une analyse statistique avec des échantillons plus importants et couvrant de façon adéquate l'ensemble des paramètres (fréquence minimale, étendue) permettrait de l'affirmer. La quantification de l'utilisation des variations abruptes de fréquences serait de nature à mettre en avant au contraire une proximité entre balearica et tyrrhenica.

\section{POURQUOI DE TELLES FRÉQUENCES CHEZ LES GOBEMOUCHES INSULAIRES?}

Au cours d'épisodes de glaciations, les populations continentales de Gobemouche gris ont trouvé un abri dans deux refuges climatiques dont on ne connaît pas précisément à ce jour la localisation, mais qui ont donné les populations actuelles qui se répartissent en deux lignées génétiques bien différenciées (Pons et al. 2016). Les îles ouest méditerranéennes ont quant à elles constitué un troisième refuge qui aurait permis la forte évolution du chant au sein de la lignée insulaire du fait de l'isolement des îles et de conditions particulières qui y règnent. Compte tenu de ce que l'on connaît de la théorie de la niche acoustique et du relâchement de caractères dans les îles (Robert et al. 2019), l'insularité est susceptible d'entraîner un élargissement de la gamme des fréquences émises et c'est ce qui s'est produit chez tyrrhenica avec l'émission de fréquences très élevées, que l'on ne s'attendrait pas à entendre d'un oiseau mais plutôt d'un insecte, voire d'un chiroptère. Notons que chez les Grylloidea (grillons), ce sont également des formes insulaires qui détiennent le record des plus hautes fréquences émises: le précédent record était détenu par Lebinthus santoensis Robillard, 2009 (Vanuatu) avec $26,5 \mathrm{kHz}$; une espèce nouvellement décrite de Nouvelle-Calédonie, Pixibinthus sonicus Anso \& Robillard, 2016, possède un chant d'appel entièrement ultrasonique avec une fréquence dominante proche de $30 \mathrm{kHz}$ (Anso et al. 2016). Rappelons que l'émission de fréquences élevées peut prédisposer certaines espèces d'oiseaux à vivre dans un habitat urbain bruyant où les communications se trouvent alors facilitées, car n'utilisant pas le même spectre de fréquence que les nuisances sonores (Hu \& Cardoso 2009). En Corse, les gobemouches vivent dans divers types d'habitats, notamment dans les villes et villages à proximité des routes et des habitations. Mais à ce stade de nos connaissances, il est impossible de connaître les facteurs à l'origine des hautes fréquences utilisées par les gobemouches de Corse et de Sardaigne. Une autre lignée insulaire (Baléares) aurait conservé une étendue de registre similaire à celle de la forme continentale, tout en utilisant des fréquences nettement plus élevées.

\section{CONCLUSION}

L'exemple des grimpereaux a permis d'illustrer la manière dont les phrases de chants sont analysées pour évaluer une divergence entre deux espèces selon les critères de Tobias et al. (2010). L'exemple des merles dyals montre des différences de gammes de fréquence utilisées mais aussi une différence de structure car si le chant est constitué de phrases bien distinctes chez C. saularis, cela n'est pas le cas chez $C$. mindanensis. Dans le cas des gobemouches, une particularité importante est l'existence de deux types de chants (chant territorial simple et chant nuptial plus complexe) qu'il a semblé préférable de distinguer dans les analyses. Par ailleurs, ces chants ne sont pas en règle générale constitués de phrases. Ils peuvent au contraire être émis de façon plus ou moins continue, ce qui rend peu pertinent le paramètre "durée moyenne». Quant aux paramètres "nombre moyen de notes" et "rythme d'émission" (nombre de notes / seconde), ils n'ont pas pu être utilisés dans l'immédiat (l'existence d'éléments très brefs et très rapprochés que l'oreille humaine est incapable de séparer mais qui peuvent néanmoins être individualisés sur sonagramme a pour conséquence une difficulté d'ordre méthodologique: doit-on ou non les considérer comme des notes distinctes?). Les analyses présentées ici ont introduit un 


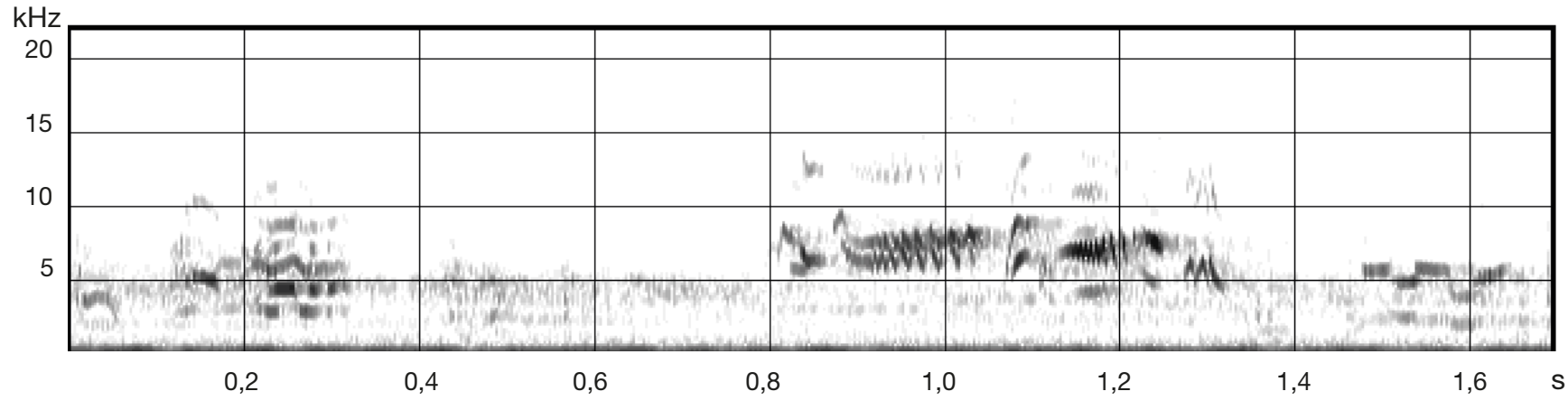

FIG. 11. - Chant nuptial de Gobemouche gris Muscicapa striata striata, Compiègne (Oise, France) enregistrement de J. Comolet-Tirman (mai 1992). Son MNHN no MNHN-SO-2017-134.

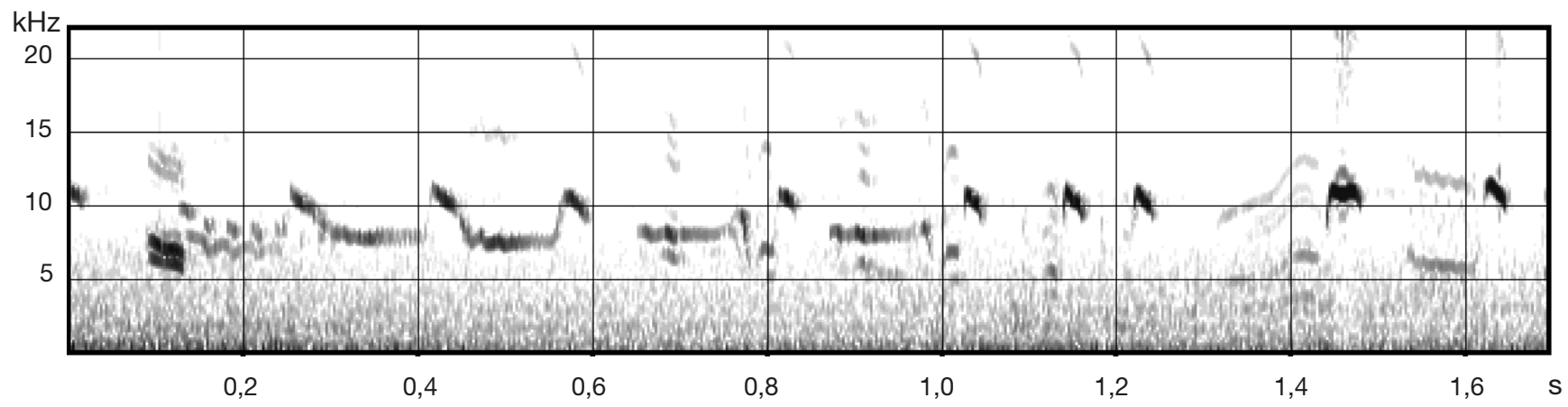

FIG. 12. - Chant nuptial de Gobemouche méditerranéen Muscicapa tyrrhenica balearica, Majorque (Baléares, Espagne) enregistrement de J. Comolet-Tirman (mai 2018). Son MNHN no MNHN-SO-2018-127.

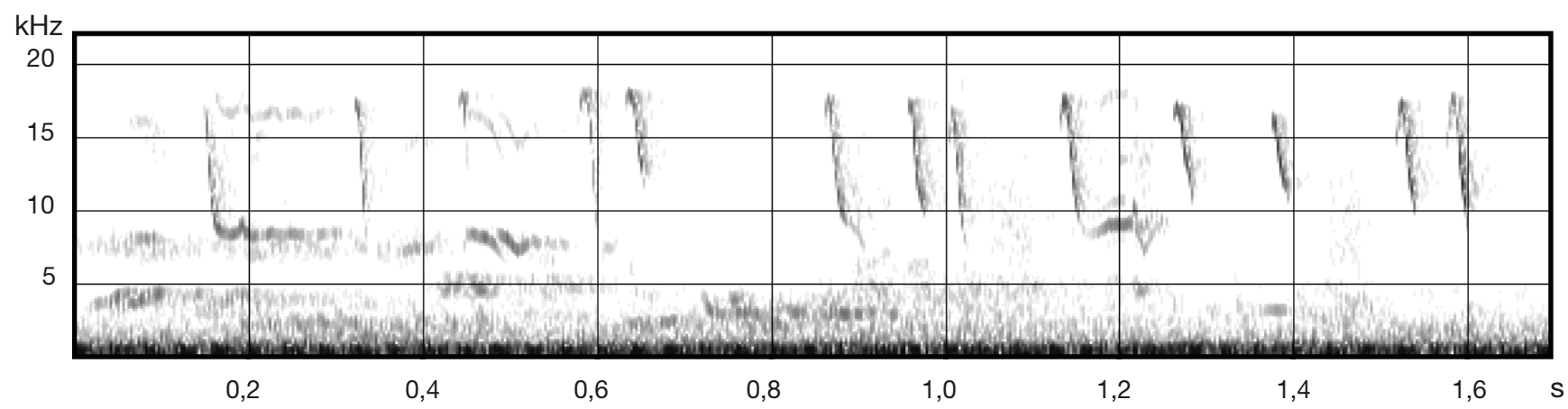

FIG. 13. - Chant nuptial de Gobemouche méditerranéen Muscicapa tyrrhenica tyrrhenica, Pietracorbara (Haute-Corse, France) enregistrement de J. ComoletTirman (mai 2017). Son MNHN no MNHN-SO-2017-110.

TABLEAU 4. - Statistique d de Cohen appliquée aux chants nuptiaux de trois formes de gobemouches Muscicapa. Les différences sont toutes exceptionnelles (d > 10) ou majeures $(d>5)$ selon la terminologie de Tobias et al. (2010).

\begin{tabular}{lcc}
\hline Paires de taxons & Cohen's d pour la fréquence maximale & Cohen's d pour la fréquence dominante \\
\hline tyr / str & 21,2 & 25,7 \\
$\mathrm{bal} / \mathrm{str}$ & 5,5 & 8,2 \\
tyr / bal & 11 & 8,9 \\
\hline
\end{tabular}

paramètre additionnel (changement brusque de fréquence) mais ont laissé de côté les trois premiers paramètres temporels. En outre, l'étude de la fréquence minimale émise par les gobemouches insulaires n'a pas toujours été possible à cause du bruit de fond présent dans certains enregistrements.
L'étendue moyenne des fréquences émises souffre donc d'un faible échantillon et, surtout dans le cas de balearica, d'une sous-estimation potentielle. Il ressort malgré tout que la divergence entre les chants nuptiaux de tyrrhenica et de striata est exceptionnelle, tendant à démontrer qu'une barrière 
comportementale est aujourd'hui possiblement à l'œuvre, empêchant les croisements de ces deux formes lorsqu'elles sont potentiellement en contact lors du retour des oiseaux à l'issue de leur hivernage africain. Sur la base de critères essentiellement morphologiques, un guide photographique récent (Shirihai \& Svensson 2018) en vient à traiter balearica comme une espèce distincte mais relègue tyrrhenica au rang de simple sous-espèce de striata. La pertinence de ces choix est questionnée par Olioso (Olioso et al. 2019). La bioacoustique montre que la forme la plus distincte sur le plan vocal est bien tyrrhenica, qui mérite à n'en pas douter un statut spécifique distinct de la forme continentale. Le traitement de balearica semble encore incertain. On pourrait hésiter en particulier entre Muscicapa tyrrhenica balearica, option suggérée par Pons et al. (2016) et retenue dans cet article, et Muscicapa balearica qui pourrait s'imposer en tenant compte des statistiques $\mathrm{d}$ de Cohen présentées ici, à nuancer par le fait que les brusques changements de fréquence constituent un caractère commun à balearica et tyrrhenica, totalement absent chez striata (Viganò et al. 2019). Une décision bien établie scientifiquement ne pourra émerger qu'après compilation détaillée des données moléculaires, morphologiques et acoustiques et approfondissement des mécanismes d'isolement reproducteur qui dépassent le cadre de cet article.

\section{Remerciements}

L'auteur remercie les preneurs de sons qui ont grandement contribué à cet article en permettant d'augmenter la taille des échantillons de chants de gobemouches étudiés, et en particulier Rob Van Bemmelen, Claude Chappuis, François Charron, Fernand Deroussen, Jérôme Fischer, Gabriel Leite et Julien Rochefort, ainsi que Frédéric Guilbert, gestionnaire de la sonothèque du Muséum national d'Histoire naturelle, et Frédéric Jiguet pour l'iconographie. Il adresse ses plus vifs remerciements aux relecteurs, Jean-Marc Pons et Stanislas Wroza, ainsi qu’à Jean-Philippe Siblet pour la photo de couverture ainsi que pour ses commentaires sur une première version de l'article. Merci également à tous les preneurs de son de la base de données en ligne XENOCANTO (https://www.xeno-canto.org, dernière consultation le 27 octobre 2019).

\section{RÉFÉRENCES}

Anso J., Barrabé L., Desutter-Grandcolas L., Jourdan H., Grandcolas P., Dong J. \& Robillard T. 2016. - Old lineage on an old island: Pixibinthus, a new Cricket genus endemic to New Caledonia shed light on gryllid diversification in a hotspot of biodiversity. PLoS ONE 11 (3): e0150920. https:// doi.org/10.1371/journal.pone. 0150920

BOESMAN P. 2016. - Notes on the vocalizations of Oriental Magpierobin (Copsychus saularis). HBW alive ornithological note 429, in Handbook of the Birds of the World Alive. Lynx Editions, Barcelone. http://www.hbw.com/node/1271524, dernière consultation le 31 juillet 2019.

Chappuis C. 1976. - Oiseaux de Corse et de Méditerranée. Disque 33 T., Alauda, supplément sonore, disque $\mathrm{n}^{\circ} 7$.

Comolet-Tirman J. 2017. - Les vocalisations du Gobemouche gris Muscicapa striata tyrrhenica en Corse: perspectives pour les décisions relatives à son statut taxonomique. Alauda 85 (4): 295-306.

COMOleT-Tirman J. 2018. - Note sur le chant nuptial du Gobemouche des Baléares Muscicapa tyrrhenica balearica à Majorque. Alauda 86 (3): 235-236.

Del Hoyo J. \& Collar N. J. 2016. - HBW and BirdLife International Illustrated Checklist of the Birds of the World. Volume 2, Passerines. Editions Lynx, Barcelone, 1013 p.

DoOling R. J. \& PRIOR N. H. 2017. - Do we hear what birds hear in birdsong? Animal Behaviour 124: 283-289. https://doi. org/10.1016/j.anbehav.2016.10.012

Hu Y. \& CARDOSO G. C. 2009. - Are bird species that vocalize at higher frequencies preadapted to inhabit noisy urban areas? Behavioral Ecology 20 (6):1268-1273. https://doi.org/10.1093/beheco/ arp131

Olioso G., Thibault J.-C., Piacentini J. \& Pons J.-M. 2019. Le Gobemouche tyrrhénien : nouvelle espèce endémique des îles de Méditerranée occidentale. Ornithos 26 (2): 67-77.

Peterson R., Mountfort G. \& Hollom P. A. D. 1971. Guide des Oiseaux d'Europe, Sème édition. Delachaux et Niestlé, Neuchatel, 447 p.

Pons J.-M., Thibault J.-C., Aymí R., Grussu M., Muntaner J., Olioso G., Sunyer J. R., Touinri M. \& Fuchs J. 2016. The role of western Mediterranean islands in the evolutionary diversification of the spotted flycatcher Muscicapa striata, a longdistance migratory passerine species. Journal of Avian Biology 47 (3): 386-398. https://doi.org/10.1111/jav.00859

POnS J.-M., FuChS J., Olioso G. \& Thibault J.-C. 2017. — Une nouvelle espèce de gobemouche en Corse. Le Courrier de la Nature 302 (mars-avril): 18-20.

Robert A., Lengagne T., Melo M., Gardette V., Julien S., Covas R., Gomez D. \& Doutrelant C. 2019. - The theory of island biogeography and soundscapes: Species diversity and the organization of acoustic communities. Journal of Biogeography 46 (9): 1-11. https://doi.org/10.1111/jbi.13611

SHIRIHAi H. \& SVENSSON L. 2018. - Handbook of Western Palearctic Birds, Volume II, Passerines: flycatchers to buntings. Christopher Helm, London, 656 p.

Sueur J. \& Boistel R. 1998. — La bioacoustique. Le Courrier de la Nature 173 (sept.-oct.): 32-37.

Tobias J. A., Seddon N., Spottiswoode C. N., Pilgrim J. D., FishPOOL L. D. \& COLlaR N. J. 2010. - Quantitative criteria for species delimitation. Ibis 152 (4): 724-746. https://doi. org/10.1111/j.1474-919X.2010.01051.x

VIGANÒ M. 2015. — Indagini bioacustiche e genetiche sul Pigliamosche tirrenico (Muscicapa striata tyrrhenica). Thèse de l'Université de Pavie, Pavie, 65 p.

Vigano M., CORSO A., Illa M. \& STARnini L. 2019. - Identification of Mediterranean Flycatcher. Dutch Birding 41: 295-317.

Wroza S. 2019. - Le Gobemouche tyrrhénien M. tyrrhenica: un exemple de vocalisation extrême. Ornithos 26 (2): 78-82. 
ANNEXES

ANNEXE 1. - Références sonores.

\begin{tabular}{|c|c|c|c|c|c|c|c|}
\hline Pays & Localité & Date & Forme & Type de chant & Référence & $\begin{array}{l}\text { Durée } \\
\text { étudiée }\end{array}$ & $\begin{array}{l}\text { Preneur de } \\
\text { son }\end{array}$ \\
\hline France & Dourdan (91) & 01/05/2010 & striata & nuptial & MNHN-SO-2020-617 & $44 \mathrm{~s}$ & J. Rochefort \\
\hline France & Compiègne (60) & 12/05/1992 & striata & nuptial & MNHN-SO-2017-134 & $60 \mathrm{~s}$ & $\begin{array}{l}\text { J. Comolet- } \\
\text { Tirman }\end{array}$ \\
\hline France & Compiègne (60) & 12/05/1992 & striata & territorial & MNHN-SO-2017-134 & $4 \mathrm{~s}$ & $\begin{array}{l}\text { J. Comolet- } \\
\text { Tirman }\end{array}$ \\
\hline France & Fontainebleau (77) & $22 / 05 / 2007$ & striata & nuptial & MNHN-SO-2020-618 & $2 \mathrm{mn} 03 \mathrm{~s}$ & J. Rochefort \\
\hline France & Fontainebleau (77) & $22 / 05 / 2007$ & striata & territorial & MNHN-SO-2020-619 & $1 \mathrm{mn} 31 \mathrm{~s}$ & J. Rochefort \\
\hline France & Fontainebleau (77) & 27/05/2013 & striata & territorial & MNHN-SO-2020-620 & $34 \mathrm{~s}$ & J. Rochefort \\
\hline France & Montigny/Loing (77) & 10/05/1992 & striata & territorial & MNHN-SO-2017-133 & $2 \mathrm{mn} 02 \mathrm{~s}$ & $\begin{array}{l}\text { J. Comolet- } \\
\text { Tirman }\end{array}$ \\
\hline France & Milly-la-Forêt (91) & 07/07/1986 & striata & territorial & $\begin{array}{l}\text { Oiseaux de } \\
\text { Fontainebleau }\end{array}$ & $32 \mathrm{~s}$ & F. Charron \\
\hline France & Arbonne-la-Forêt (77) & 27/05/1992 & striata & territorial & MNHN-SO-2016-7999 & $27 \mathrm{~s}$ & F. Deroussen \\
\hline France & Forêt de Villefermoy (77) & 01/06/1996 & striata & territorial & MNHN-SO-2016-8003 & $30 \mathrm{~s}$ & F. Deroussen \\
\hline France & St Léger la Montagne (87) & $19 / 05 / 2005$ & striata & territorial & MNHN-SO-2016-8004 & $30 \mathrm{~s}$ & F. Deroussen \\
\hline France/Corse & Aleria (2B) & $13 / 05 / 2017$ & tyrrhenica & nuptial & MNHN-SO-2017-111 & $13 \mathrm{~s}$ & $\begin{array}{l}\text { J. Comolet- } \\
\text { Tirman }\end{array}$ \\
\hline France/Corse & Aleria (2B) & $13 / 05 / 2017$ & tyrrhenica & territorial & MNHN-SO-2017-112 & $4 \mathrm{mn}$ & $\begin{array}{l}\text { J. Comolet- } \\
\text { Tirman }\end{array}$ \\
\hline France/Corse & Pietracorbara (2B) & 09/05/2017 & tyrrhenica & nuptial & MNHN-SO-2017-110 & $16 s$ & $\begin{array}{l}\text { J. Comolet- } \\
\text { Tirman }\end{array}$ \\
\hline France/Corse & Pietracorbara (2B) & 09/05/2017 & tyrrhenica & territorial & MNHN-SO-2017-109 & $3 \mathrm{mn}$ & $\begin{array}{l}\text { J. Comolet- } \\
\text { Tirman }\end{array}$ \\
\hline France/Corse & Tiuccia (2A) & $17 / 05 / 2017$ & tyrrhenica & nuptial & MNHN-SO-2017-113 & $40 \mathrm{~s}$ & $\begin{array}{l}\text { J. Comolet- } \\
\text { Tirman }\end{array}$ \\
\hline France/Corse & Tiuccia (2A) & $18 / 05 / 2017$ & tyrrhenica & territorial & MNHN-SO-2017-123 & $35 \mathrm{~s}$ & $\begin{array}{l}\text { J. Comolet- } \\
\text { Tirman }\end{array}$ \\
\hline France/Corse & Olmeto $(2 \mathrm{~A})$ & 07/05/2018 & tyrrhenica & nuptial & XC428243 & $1 \mathrm{mn} 54 \mathrm{~s}$ & $\begin{array}{l}\text { R. Van } \\
\text { Bemmelen }\end{array}$ \\
\hline $\begin{array}{l}\text { Italie/ } \\
\quad \text { Sardaigne }\end{array}$ & Carloforte & $11 / 05 / 2017$ & tyrrhenica & territorial & XC370713 & $55 \mathrm{~s}$ & J. Fischer \\
\hline $\begin{array}{l}\text { Espagne/ } \\
\text { Majorque }\end{array}$ & Muro & $14 / 05 / 2018$ & balearica & nuptial & MNHN-SO-2018-124 & $4,4 \mathrm{~s}$ & $\begin{array}{l}\text { J. Comolet- } \\
\text { Tirman }\end{array}$ \\
\hline $\begin{array}{l}\text { Espagne/ } \\
\text { Majorque }\end{array}$ & Playa de Muro & $15 / 05 / 2018$ & balearica & nuptial & MNHN-SO-2018-127 & $11 \mathrm{~s}$ & $\begin{array}{l}\text { J. Comolet- } \\
\text { Tirman }\end{array}$ \\
\hline $\begin{array}{l}\text { Espagne/ } \\
\text { Majorque }\end{array}$ & Port de Pollença & $14 / 05 / 2018$ & balearica & nuptial & MNHN-SO-2018-126 & $2 \mathrm{~s}$ & $\begin{array}{l}\text { J. Comolet- } \\
\text { Tirman }\end{array}$ \\
\hline $\begin{array}{l}\text { Espagne/ } \\
\text { Majorque }\end{array}$ & S'Albufera & $12 / 05 / 2018$ & balearica & territorial & MNHN-SO-2018-123 & $6 s$ & $\begin{array}{l}\text { J. Comolet- } \\
\text { Tirman }\end{array}$ \\
\hline $\begin{array}{l}\text { Espagne/ } \\
\text { Majorque }\end{array}$ & NE Majorque & 06/1975 & balearica & territorial & $\begin{array}{l}\text { Alauda suppl. sonore } \\
\text { no } 7\end{array}$ & $40 \mathrm{~s}$ & C. Chappuis \\
\hline $\begin{array}{l}\text { Espagne/ } \\
\text { Majorque }\end{array}$ & Serra de Tramuntana & $19 / 06 / 2017$ & balearica & territorial & XC376589 & $12,5 \mathrm{~s}$ & G. Leite \\
\hline
\end{tabular}


ANNEXE 2. - Fiches synthétiques des gobemouches.

\begin{tabular}{|c|c|c|c|c|c|c|}
\hline & & Principaux paramètres & $\begin{array}{c}\text { Fréquence } \\
\text { maximale }\end{array}$ & $\begin{array}{l}\text { Fréquence } \\
\text { dominante }\end{array}$ & $\begin{array}{c}\text { Fréquence } \\
\text { minimale }\end{array}$ & $\begin{array}{l}\text { Étendue } \\
\text { moyenne }\end{array}$ \\
\hline \multirow[t]{8}{*}{ Continent } & \multirow[t]{4}{*}{ Nuptial } & $\begin{array}{l}\text { Dourdan } \\
\text { Compiègne }\end{array}$ & $\begin{array}{l}10710 \\
10250\end{array}$ & $\begin{array}{l}7246 \\
7778\end{array}$ & $\begin{array}{l}1920 \\
1150\end{array}$ & $\begin{array}{l}8790 \\
9100\end{array}$ \\
\hline & & Fontainebleau & 10075 & 7190 & 1400 & 8675 \\
\hline & & Valeurs moyennes en $\mathrm{Hz}$ & 10345 & 7405 & 1490 & 8855 \\
\hline & & Écart type & 328 & 325 & 393 & 220 \\
\hline & \multirow[t]{2}{*}{ Territorial } & $\begin{array}{l}\text { Compiègne } \\
\text { Fontainebleau - parcelle } 604 \\
\text { Fontainebleau - Feuillards } \\
\text { Montigny-sur-Loing } \\
\text { Coquibus } \\
\text { Chanfroy } \\
\text { Villefermoy } \\
\text { Tourbière des Dauges }\end{array}$ & $\begin{array}{r}9000 \\
10500 \\
9500 \\
10000 \\
9500 \\
9200 \\
9900 \\
10050 \\
\end{array}$ & $\begin{array}{l}7163 \\
7555 \\
7487 \\
7536 \\
7254 \\
7284 \\
7412 \\
6840 \\
\end{array}$ & $\begin{array}{l}4500 \\
3900 \\
2250 \\
4500 \\
5000 \\
1800 \\
2800 \\
4250 \\
\end{array}$ & $\begin{array}{l}4500 \\
6600 \\
7250 \\
5500 \\
4500 \\
7400 \\
7100 \\
5800\end{array}$ \\
\hline & & $\begin{array}{l}\text { Valeurs moyennes en } \mathrm{Hz} \\
\text { Écart type }\end{array}$ & $\begin{array}{r}9706 \\
495\end{array}$ & $\begin{array}{r}7316 \\
239\end{array}$ & $\begin{array}{l}3625 \\
1183\end{array}$ & $\begin{array}{l}6081 \\
1185\end{array}$ \\
\hline & \multirow{2}{*}{$\begin{array}{r}\text { Cumul (nuptial } \\
\text { et territorial) }\end{array}$} & Valeurs moyennes en $\mathrm{Hz}$ & 9880 & 7340 & 3043 & 6838 \\
\hline & & Écart type & 531 & 250 & 1416 & 1634 \\
\hline \multirow[t]{5}{*}{$\begin{array}{l}\text { Corse- } \\
\text { Sardaigne }\end{array}$} & \multirow[t]{2}{*}{ Nuptial } & $\begin{array}{l}\text { Aleria } \\
\text { Pietracorbara } \\
\text { Tiuccia } \\
\text { Olmeto }\end{array}$ & $\begin{array}{l}19000 \\
18500 \\
18000 \\
17000\end{array}$ & $\begin{array}{l}17881 \\
17452 \\
16876 \\
14797\end{array}$ & $\begin{array}{c}3000 \\
3100 \\
2070 \\
-\end{array}$ & $\begin{array}{c}16000 \\
15400 \\
15930 \\
-\end{array}$ \\
\hline & & $\begin{array}{l}\text { Valeurs moyennes en } \mathrm{Hz} \\
\text { Écart type }\end{array}$ & $\begin{array}{r}18125 \\
854\end{array}$ & $\begin{array}{r}16752 \\
1367\end{array}$ & $\begin{array}{r}2723 \\
568\end{array}$ & $\begin{array}{r}15777 \\
328\end{array}$ \\
\hline & \multirow[t]{2}{*}{ Territorial } & $\begin{array}{l}\text { Aleria } \\
\text { Pietracorbara } \\
\text { Tiuccia } \\
\text { Carloforte (Sardaigne) }\end{array}$ & $\begin{array}{l}20000 \\
18900 \\
17750 \\
15800\end{array}$ & $\begin{array}{l}7616 \\
6848 \\
7057 \\
7066\end{array}$ & $\begin{array}{l}5000 \\
5500 \\
5000 \\
4050\end{array}$ & $\begin{array}{l}15000 \\
13400 \\
12750 \\
11750\end{array}$ \\
\hline & & $\begin{array}{l}\text { Valeurs moyennes en } \mathrm{Hz} \\
\text { Écart type }\end{array}$ & $\begin{array}{l}18113 \\
17955\end{array}$ & $\begin{array}{r}7147 \\
329 \\
\end{array}$ & $\begin{array}{r}4888 \\
606 \\
\end{array}$ & $\begin{array}{r}13225 \\
1364 \\
\end{array}$ \\
\hline & $\begin{array}{r}\text { Cumul (nuptial } \\
\text { et territorial) }\end{array}$ & $\begin{array}{l}\text { Valeurs moyennes en } \mathrm{Hz} \\
\text { Écart type }\end{array}$ & $\begin{array}{r}18119 \\
1301\end{array}$ & $\begin{array}{r}11949 \\
5216\end{array}$ & $\begin{array}{l}3960 \\
1276\end{array}$ & $\begin{array}{r}14319 \\
1681\end{array}$ \\
\hline \multirow[t]{5}{*}{ Baléares } & \multirow[t]{2}{*}{ Nuptial } & $\begin{array}{l}\text { Playa de Muro } \\
\text { Port de Pollença } \\
\text { Muro }\end{array}$ & $\begin{array}{l}12250 \\
12375 \\
13500\end{array}$ & $\begin{array}{l}10807 \\
11514 \\
12559\end{array}$ & $\begin{array}{c}2750 \\
6900 \\
- \\
\end{array}$ & $\begin{array}{c}9500 \\
5475 \\
- \\
\end{array}$ \\
\hline & & $\begin{array}{l}\text { Valeurs moyennes en } \mathrm{Hz} \\
\text { Écart type }\end{array}$ & $\begin{array}{r}12708 \\
688\end{array}$ & $\begin{array}{r}11627 \\
881\end{array}$ & $\begin{array}{l}4825 \\
2934\end{array}$ & $\begin{array}{l}7488 \\
2846\end{array}$ \\
\hline & \multirow[t]{2}{*}{ Territorial } & $\begin{array}{l}\text { S'Albufera } \\
\text { Nord Est Majorque } \\
\text { Serra de Tramuntana }\end{array}$ & $\begin{array}{l}10100 \\
12500 \\
13700\end{array}$ & $\begin{array}{l}7093 \\
6592 \\
7582\end{array}$ & $\begin{array}{l}6000 \\
6000 \\
6200\end{array}$ & $\begin{array}{l}4100 \\
6500 \\
7500\end{array}$ \\
\hline & & $\begin{array}{l}\text { Valeurs moyennes en } \mathrm{Hz} \\
\text { Écart type }\end{array}$ & $\begin{array}{r}12100 \\
1833\end{array}$ & $\begin{array}{r}7089 \\
495\end{array}$ & $\begin{array}{r}6067 \\
115\end{array}$ & $\begin{array}{l}6033 \\
1747\end{array}$ \\
\hline & $\begin{array}{l}\text { Cumul (nuptial } \\
\text { et territorial) }\end{array}$ & $\begin{array}{l}\text { Valeurs moyennes en } \mathrm{Hz} \\
\text { Écart type }\end{array}$ & $\begin{array}{r}12404 \\
1282\end{array}$ & $\begin{array}{l}9358 \\
2566\end{array}$ & $\begin{array}{l}5570 \\
1619\end{array}$ & $\begin{array}{l}6615 \\
2046\end{array}$ \\
\hline
\end{tabular}


ANNEXE 3. - Cartes d'identité sonores individuelles des gobemouches Muscicapa par type de chant.

\section{Référence MNHN-SO-2020-617}

TYPE. Continental

DATE. 01/05/2010 à $12 \mathrm{~h} 53$

DURÉE ANALYSÉE. $44 \mathrm{~s}$

LoCALITÉ. Forêt de Dourdan (Essonne)

Type DE CHANT. Nuptial

Preneur DE SON. Julien ROCHEFORT

\begin{tabular}{lcc}
\hline Principaux paramètres & Valeurs en Hz & Notes \\
\hline Fréquence dominante & 7246 & A8 \\
Fréquence maximale & 10710 & E9 \\
Fréquence minimale & 1920 & B6 \\
Étendue & 8790 & - \\
\hline
\end{tabular}

Référence MNHN-SO-2017-134 [restreint à 60s, entre 4s et 64s]

TYPE. Continental

DATE. $12 / 05 / 1992$

DURÉE ANALYSÉE. 60s

LOCALITÉ. Forêt de Compiègne (Oise)

TyPe DE CHANT. Nuptial

Preneur DE SON. Jacques COMOLET-TIRMAN

\begin{tabular}{lcc}
\hline Principaux paramètres & Valeurs en Hz & Notes \\
\hline Fréquence dominante & 7778 & B8 \\
Fréquence maximale & 10250 & D\#9/E9 \\
Fréquence minimale & 1150 & D6 \\
Étendue & 9100 & - \\
\hline
\end{tabular}

Référence MNHN-SO-2017-134 [restreint à 4s, entre 0 et $4 \mathrm{~s}$ ]

TYPE. Continental

DATE. $12 / 05 / 1992$

DURÉE ANALYSÉE. $4 \mathrm{~s}$

LOCALITÉ. Forêt de Compiègne (Oise)

Type DE CHANT. Territorial

PRENEUR DE SON. Jacques COMOLET-TIRMAN

\begin{tabular}{lcc}
\hline Principaux paramètres & Valeurs en Hz & Notes \\
\hline Fréquence dominante & 7163 & A8 \\
Fréquence maximale & 9000 & - \\
Fréquence minimale & 4500 & - \\
Étendue & 4500 & - \\
\hline
\end{tabular}

Référence MNHN-SO-2020-618

TyPE. Continental

DATE. 22/05/2007 à 12 h02

DuRÉE ANALYSÉE. $2 \mathrm{mn} 03 \mathrm{~s}$

LOCALITÉ. Forêt de Fontainebleau, parcelle 604 (Seine-et-Marne)

Type DE CHANT. Nuptial

PrENEUR DE SON. Julien ROCHEFORT

\begin{tabular}{lcc}
\hline Principaux paramètres & Valeurs en Hz & Notes \\
\hline Fréquence dominante & 7190 & $\mathrm{~A} 8$ \\
Fréquence maximale & 10075 & $\mathrm{D \# 9}$ \\
Fréquence minimale & 1400 & $\mathrm{~F} 6$ \\
Étendue & 8675 & - \\
\hline
\end{tabular}


Référence MNHN-SO-2020-619

TYPE. Continental

DATE. 22/05/2007 à $11 \mathrm{~h} 59$

DurÉE ANALYSÉE. $1 \mathrm{mn} 31 \mathrm{~s}$

LOCALITÉ. Forêt de Fontainebleau, parcelle 604 (Seine-et-Marne)

Type DE CHANT. Territorial

Preneur DE SON. Julien ROCHEFORT

\begin{tabular}{lcc}
\hline Principaux paramètres & Valeurs en Hz & Notes \\
\hline Fréquence dominante & 7555 & A\#8 \\
Fréquence maximale & 10500 & - \\
Fréquence minimale & 3900 & - \\
Étendue & 6600 & - \\
\hline
\end{tabular}

Référence MNHN-SO-2020-620

TYPe. Continental

DATE. 27/05/2013

DURÉE ANALYSÉE. $34 \mathrm{~s}$

LoCALITÉ. Forêt de Fontainebleau, Feuillards (Seine-et-Marne)

TyPe DE CHANT. Territorial

PrENEUR DE SON. Julien ROCHEFORT

\begin{tabular}{lcc}
\hline Principaux paramètres & Valeurs en $\mathbf{H z}$ & Notes \\
\hline Fréquence dominante & 7487 & A\#8 \\
Fréquence maximale & 9500 & - \\
Fréquence minimale & 2250 & - \\
Étendue & 7250 & - \\
\hline
\end{tabular}

Référence MNHN-SO-2017-133

TyPe. Continental

DATE. 10/05/1992

DURÉE ANALYSÉE. $2 \mathrm{mn} 02 \mathrm{~s}$

LOCALITÉ. Montigny-sur-Loing (Seine-et-Marne)

TyPE DE CHANT. Territorial

Preneur de SON. Jacques COMOLET-TIRMAN

\begin{tabular}{lcc}
\hline Principaux paramètres & Valeurs en $\mathbf{~ H z}$ & Notes \\
\hline Fréquence dominante & 7536 & A\#8 \\
Fréquence maximale & 10000 & - \\
Fréquence minimale & 4500 & - \\
Étendue & 5500 & - \\
\hline
\end{tabular}

Référence «Oiseaux de Fontainebleau» (cassette audio)

TyPE. Continental

DATE. 07/07/1986

DuRÉE ANALYSÉE. $32 \mathrm{~s}$

LoCAlité. Le Coquibus en Forêt des Trois Pignons, Milly-la-Forêt (Essonne)

TYPE DE CHANT. Territorial

Preneur DE SON. François CHARRON

\begin{tabular}{lcc}
\hline Principaux paramètres & Valeurs en Hz & Notes \\
\hline Fréquence dominante & 7254 & A\#8 \\
Fréquence maximale & 9500 & - \\
Fréquence minimale & 5000 & - \\
Étendue & 4500 & - \\
\hline
\end{tabular}




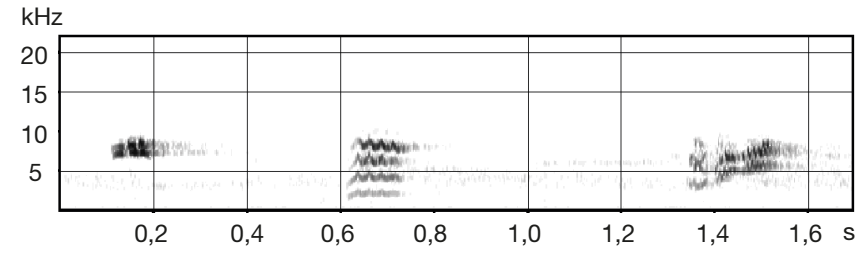

Chant territorial de Gobemouche gris Muscicapa striata striata enregistré par François Charron.

Source. Charron F. \& Comolet-Tirman J. 1993. - Oiseaux de Fontainebleau (cassette audio, publiée avec le concours de l'Association des Naturalistes de la Vallée du Loing et du massif de Fontainebleau). C.N.A.I., Paris.

\section{Référence MNHN-SO-2016-7999}

TYPE. Continental

DATE. 27/05/1992

DURÉE ANALYSÉE. $27 \mathrm{~s}$

LoCALité. Chanfroy en Forêt des Trois Pignons, Arbonne-la-Forêt (Seine-et-Marne)

TYPE DE CHANT. Territorial

PRENEUR DE SON. Fernand DEROUSSEN

\begin{tabular}{lcc}
\hline Principaux paramètres & Valeurs en $\mathbf{H z}$ & Notes \\
\hline Fréquence dominante & 7284 & A\#8 \\
Fréquence maximale & 9200 & - \\
Fréquence minimale & 1800 & - \\
Étendue & 7400 & - \\
\hline
\end{tabular}

Étendue

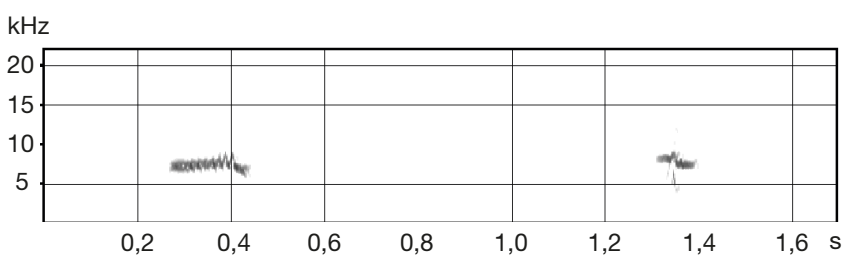

Chant territorial de Gobemouche gris Muscicapa striata striata enregistré par F. Deroussen.

\section{Référence MNHN-SO-2016-8003}

TYPE. Continental

DATE. 01/06/1996

DURÉE ANALYSÉE. 30s

LOCALITÉ. Forêt de Villefermoy (Seine-et-Marne)

TYPE DE CHANT. Territorial

Preneur DE SON. Fernand DEROUSSEN

\section{Principaux paramètres}

Valeurs en $\mathrm{Hz}$

Fréquence dominante

Fréquence maximale

Fréquence minimale

Étendue

\section{2}

9900

2800

7100
Notes

A\#8

$-$

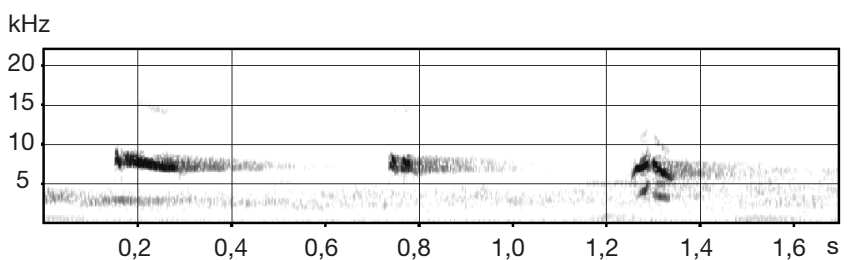

Chant territorial de Gobemouche gris Muscicapa striata striata enregistré par F. Deroussen. 
Référence MNHN-SO-2016-8004

TYPE. Continental

DATE. 19/05/2005

DURÉE ANALYSÉE. 30s

LoCAlité. Tourbière des Dauges, St Léger la Montagne (Haute-Vienne)

TYPE DE CHANT. Territorial

PRENEUR DE SON. Fernand DEROUSSEN

\begin{tabular}{lcc}
\hline Principaux paramètres & Valeurs en Hz & Notes \\
\hline Fréquence dominante & 6840 & A8 \\
Fréquence maximale & 10050 & - \\
Fréquence minimale & 4250 & - \\
Étendue & 5800 & - \\
\hline
\end{tabular}

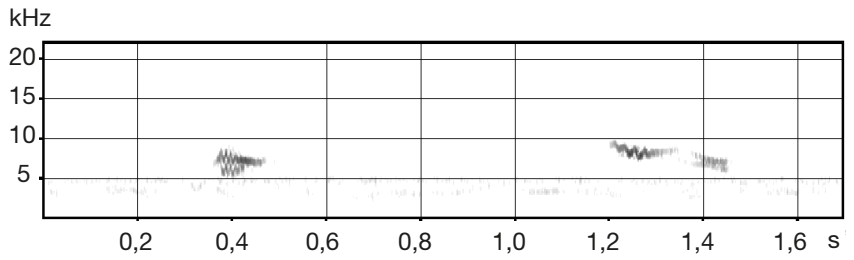

Chant territorial de Gobemouche gris Muscicapa striata striata enregistré par F. Deroussen.

Référence MNHN-SO-2017-111 [55s restreint à 13s, entre 5,4s et 18,4s]

TYPE. Tyrrhénien (Corse)

DATE. 13/05/2017

DURÉE ANALYSÉE. $13 \mathrm{~s}$

LOCALITÉ. Aleria (Haute-Corse)

TyPE DE CHANT. Nuptial (mais la séquence complète est mixte nuptial/territorial)

Preneur de son. Jacques COMOLET-TIRMAN

\begin{tabular}{lcc}
\hline Principaux paramètres & Valeurs en Hz & Notes \\
\hline Fréquence dominante & 17881 & C\#10 \\
Fréquence maximale & 19000 & - \\
Fréquence minimale & 3000 environ & - \\
Étendue & 16000 environ & - \\
\hline
\end{tabular}

Référence MNHN-SO-2017-110 [4mn 23s restreint à 16s, entre 42s et 58s]

TYPE. Tyrrhénien (Corse)

DATE. 09/05/2017

DuRÉE ANALYSÉE. $16 \mathrm{~s}$

LOCALITÉ. Pietracorbara (Haute-Corse)

TyPE DE CHANT. Nuptial (mais la séquence complète est mixte nuptial/territorial)

PRENEUR DE SON. Jacques COMOLET-TIRMAN

Principaux paramètres

Fréquence dominante

Fréquence maximale

Fréquence minimale

Étendue

Valeurs en $\mathbf{H z}$
17452
18500
3100
15400 environ

Notes
C\#10
-
-
-


Référence MNHN-SO-2017-113 [6mn 05s restreint à 40s, approximativement entre 151s et 187s]

TYPE. Tyrrhénien (Corse)

DATE. 17/05/2017

DURÉE ANALYSÉE. 40s

LOCALITÉ. Tiuccia (Corse-du-Sud)

Type DE Chant. Nuptial

Preneur de SON. Jacques COMOLET-TIRMAN

\begin{tabular}{lcc}
\hline Principaux paramètres & Valeurs en $\mathbf{H z}$ & Notes \\
\hline Fréquence dominante & 16876 & $\mathrm{C} 10$ \\
Fréquence maximale & 18000 & - \\
Fréquence minimale & 2070 & $\mathrm{C} 7$ \\
Étendue & 15930 sous réserve & - \\
\hline
\end{tabular}

\section{Référence XC428243}

TYPE. Tyrrhénien (Corse)

DATE. 07/05/2018 à $7 \mathrm{~h}$

DURÉE ANALYSÉE. $1 \mathrm{mn} 54 \mathrm{~s}$

LOCALITÉ. Olmeto (Corse-du-Sud)

Altitude. $70 \mathrm{~m}$

Type DE CHANT. Nuptial

PRENEUR DE SON. Rob van BEMMELEN

\begin{tabular}{lcc}
\hline Principaux paramètres & Valeurs en $\mathbf{~ H z}$ & Notes \\
\hline Fréquence dominante & 14797 & A\#9 \\
Fréquence maximale & 17000 & - \\
Fréquence minimale & Impossible à déterminer & - \\
Étendue & Impossible à déterminer & - \\
\hline
\end{tabular}

Référence MNHN-SO-2017-112 [5mn 01s restreint entre 28s et 4mn25s]

TYPE. Tyrrhénien (Corse)

DATE. 13/05/2017

DURÉE ANALYSÉE. $3 \mathrm{mn} 57 \mathrm{~s}$

LOCALITÉ. Aleria (Haute-Corse)

TYPE DE CHANT. Territorial

PRENEUR DE SON. Jacques COMOLET-TIRMAN

\begin{tabular}{lcc}
\hline Principaux paramètres & Valeurs en Hz & Notes \\
\hline Fréquence dominante & 7616 & A\#8 \\
Fréquence maximale & 20000 & - \\
Fréquence minimale & 5000 & - \\
Étendue & 15000 & - \\
\hline
\end{tabular}

REMARQUE. Maximum le plus souvent vers 18000.

Référence MNHN-SO-2017-109 [5mn 03s analysé sur séquence spéciale expurgée des alarmes et de chants de bouscarle]

TYPE. Tyrrhénien (Corse)

DATE. 09/05/2017

DuRÉE ANALYSÉE. Près de $3 \mathrm{mn}$

LOCALITÉ. Pietracorbara (Haute-Corse)

TYPE DE CHANT. Territorial

Preneur de SON. Jacques COMOLET-TIRMAN

\begin{tabular}{lcc}
\hline Principaux paramètres & Valeurs en Hz & Notes \\
\hline Fréquence dominante & 6848 & A8 \\
Fréquence maximale & 18900 & - \\
Fréquence minimale & 5500 & - \\
Étendue & 13400 & - \\
\hline
\end{tabular}


Référence MNHN-SO-2017-123

TYPE. Tyrrhénien (Corse)

DATE. 18/05/2017 à 7h11

DuréE ANALYSÉE. $35 \mathrm{~s}$

LOCALITÉ. Tiuccia (Corse-du-Sud)

TyPe DE CHANT. Territorial

Preneur de SON. Jacques COMOLET-TIRMAN

\begin{tabular}{lcc}
\hline Principaux paramètres & Valeurs en Hz & Notes \\
\hline Fréquence dominante & 7057 & A8 \\
Fréquence maximale & 17750 & - \\
Fréquence minimale & 5000 & - \\
Étendue & 12750 & - \\
\hline
\end{tabular}

REMARQUE. Minimales à 5500 vers 7,75s et même 5000 vers 10,3 s ou vers 24,3s

\section{Référence XC370713}

TYPE. Tyrrhénien (Sardaigne)

DATE. 11/05/2017

DuréE ANALYSÉE 55,3s

LOCALITÉ. Carloforte (Sardaigne)

TyPe DE CHANT. Territorial

PReneur De SON. Jérôme FISCHER

\begin{tabular}{lcc}
\hline Principaux paramètres & Valeurs en Hz & Notes \\
\hline Fréquence dominante & 7066 & A8 \\
Fréquence maximale & 15800 & - \\
Fréquence minimale & 4050 & - \\
Étendue & 11750 & - \\
\hline
\end{tabular}

Référence MNHN-SO-2018-124 [40s restreint à 4,4s entre 16,4s et 20,8s]

TYPE. Baléares

DATE. 14/05/2018 à 6h21

DURÉE ANALYSÉE. $4,4 \mathrm{~s}$

LOCALITÉ. Muro (Majorque)

Type DE Chant. Nuptial

Preneur DE SON. Jacques COMOLET-TIRMAN

\begin{tabular}{lcc}
\hline Principaux paramètres & Valeurs en Hz & Notes \\
\hline Fréquence dominante & 12559 & G9 \\
Fréquence maximale & 13500 & G\#9 \\
Fréquence minimale & Impossible à déterminer & - \\
Étendue & Impossible à déterminer & - \\
\hline
\end{tabular}

Référence MNHN-SO-2018-127 [1mn 54s restreint à 11s entre 35,2s et 46,2s]

TYPE. Baléares

DATE. 15/05/2018 à 9h24

DURÉE ANALYSÉE. $11 \mathrm{~s}$

LOCALITÉ. Playa de Muro (Majorque)

Type De Chant. Nuptial

Preneur DE SON. Jacques COMOLET-TIRMAN

\begin{tabular}{lcc}
\hline Principaux paramètres & Valeurs en Hz & Notes \\
\hline Fréquence dominante & 10807 & E9 \\
Fréquence maximale & 12250 & - \\
Fréquence minimale & 2750 & - \\
Étendue & 9500 & - \\
\hline
\end{tabular}


ANNEXE 3. - Suite.

REMARQUe. Un son de l'ordre de $2300 \mathrm{~Hz}$, présent dans cet enregistrement, n’a pas pu être attribué à l'espèce avec une certitude suffisante et n’a donc pas été retenu. Malgré tout, la fréquence minimale de $2750 \mathrm{~Hz}$ est donnée sous réserve.

Référence MNHN-SO-2018-126 [1mn 22s restreint à 1,6s entre 6,2s et 7,8s]

TyPE. Baléares

DATE. $14 / 05 / 2018$ à 8 h33

DurÉE ANALYSÉE. 1,6s

LOCALiTÉ. Port de Pollença (Majorque)

TyPE DE CHANT. Nuptial

Preneur de SON. Jacques COMOLET-TIRMAN

\begin{tabular}{lcc}
\hline Principaux paramètres & Valeurs en $\mathbf{~ H z}$ & Notes \\
\hline Fréquence dominante & 11514 & F\#9 \\
Fréquence maximale & 12375 & - \\
Fréquence minimale & 6900 & - \\
Étendue & 5475 & - \\
\hline
\end{tabular}

REMARQUE. Parmi les chants nuptiaux de balearica enregistrés par l'auteur, cet individu est le seul à émettre des sons passant brusquement d'environ 7 à $12 \mathrm{kHz}$ (écart > $4 \mathrm{kHz}$ ). Les paramètres mesurés et en particulier la fréquence minimale sont donnés sous réserve compte tenu de la brièveté de la séquence étudiée.

Référence MNHN-SO-2018-123 [46s restreint entre 14s et 20s]

TYPE. Baléares

DATE. 12/05/2018 à $11 \mathrm{~h} 29$

DURÉE ANALYSÉE. $6 \mathrm{~s}$

LOCALITÉ. S’Albufera (Majorque)

TYPE DE CHANT. Territorial

Preneur de SON. Jacques COMOLET-TIRMAN

\begin{tabular}{lcc}
\hline Principaux paramètres & Valeurs en $\mathbf{H z}$ & Notes \\
\hline Fréquence dominante & 7093 & A8 \\
Fréquence maximale & 10100 & - \\
Fréquence minimale & 6000 & - \\
Étendue & 4100 & - \\
\hline
\end{tabular}

Référence «Oiseaux de Corse et de Méditerranée» (disque 33 tours)

TYPE. Baléares

DATE. 06/1975

DurÉE ANALYSÉE. 40s

LOCALITÉ. N. E. Majorque

TYPE DE CHANT. Territorial

Preneur DE SON. Claude CHAPPUIS

\begin{tabular}{lcc}
\hline Principaux paramètres & Valeurs en Hz & Notes \\
\hline Fréquence dominante & 6592 & G\#8 \\
Fréquence maximale & 12500 & - \\
Fréquence minimale & 6000 & - \\
Étendue & 6500 & - \\
\hline
\end{tabular}

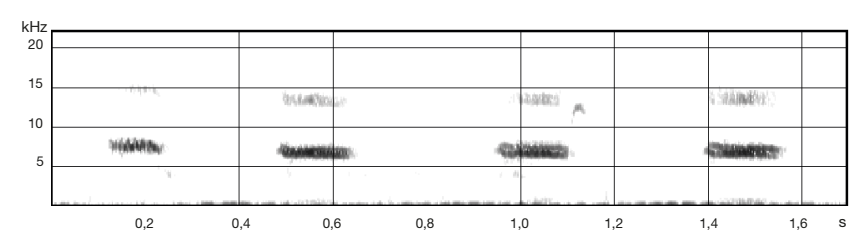

Chant territorial de Gobemouche méditerranéen Muscicapa tyrrhenica balearica enregistré par C. Chappuis.

SourCE. Chappuis C. 1976. - Oiseaux de Corse et de Méditerranée. Disque 33 T., Alauda, supplément sonore, disque $n^{\circ} 7$. 


\section{Référence XC376589}

TyPE. Baléares

DATE. 19/06/2017

DuRÉE ANALYSÉE. $12,5 \mathrm{~s}$

LoCALITÉ. Serra de Tramuntana (proche de Escorca), Majorque

ALTITUDE. $750 \mathrm{~m}$

TYPE DE CHANT. Territorial

Preneur DE SON. Gabriel LEITE

\section{Principaux paramètres}

Fréquence dominante

Fréquence maximale

Fréquence minimale

Étendue
Valeurs en $\mathrm{Hz}$

7582

13700

6200

7500
Notes

A\#8 